\title{
Are long chain acyl CoAs responsible for suppression of mitochondrial metabolism in hibernating 13-lined ground squirrels?
}

Alex Cooper, The University of Western Ontario

Supervisor: Dr. Jim Staples, The University of Western Ontario

A thesis submitted in partial fulfillment of the requirements for the Master of Science degree in Biology

(C) Alex Cooper 2013

Follow this and additional works at: https://ir.lib.uwo.ca/etd

Part of the Biology Commons

\section{Recommended Citation}

Cooper, Alex, "Are long chain acyl CoAs responsible for suppression of mitochondrial metabolism in hibernating 13-lined ground squirrels?" (2013). Electronic Thesis and Dissertation Repository. 1372.

https://ir.lib.uwo.ca/etd/1372

This Dissertation/Thesis is brought to you for free and open access by Scholarship@Western. It has been accepted for inclusion in Electronic Thesis and Dissertation Repository by an authorized administrator of Scholarship@Western. For more information, please contact wlswadmin@uwo.ca. 


\title{
ARE LONG CHAIN ACYL COAS RESPONSIBLE FOR SUPPRESSION OF MITOCHONDRIAL METABOLISM IN HIBERNATING 13-LINED GROUND SQUIRRELS?
}

(Thesis format: monograph)

by

\author{
Alex Nicole Cooper \\ Graduate Program in Biology \\ A thesis submitted in partial fulfillment \\ of the requirements for the degree of \\ Master of Science
}

The School of Graduate and Postdoctoral Studies The University of Western Ontario

London, Ontario, Canada

(C) Alex N. Cooper 2013 


\begin{abstract}
I found $44 \%$ suppression of succinate-fuelled liver mitochondrial respiration in torpid 13-lined ground squirrels compared to interbout euthermia (IBE). Palmitoyl CoA, predicted to suppress respiration by inhibiting succinate transport at the dicarboxylate transporter $(\mathrm{DCT})$, reduced respiration by $\sim 70 \%$, while butylmalonate, a known inhibitor of the DCT, only inhibited respiration by $\sim 40 \%$. In both cases inhibition of respiration proportionally affected both torpid and IBE mitochondria, suggesting that the DCT is likely not already inhibited in torpid mitochondria. The addition of carnitine, predicted to reverse suppression by facilitating transport of palmitoyl CoA into the mitochondrial matrix, had no rescuing effect on the respiration rates of mitochondria treated with palmitoyl CoA, nor did it increase the respiration rate of torpid mitochondria. Though palmitoyl CoA inhibits succinate-fuelled respiration, suppression may not be exclusively related to inhibition of succinate transport at the DCT, and is likely inhibiting additional mitochondrial transporters such as the adenine-nucleotide transporter.
\end{abstract}

Key Words: Mitochondria, Hibernation, Dicarboxylate Transporter, Long-chain acyl CoA, Palmitoyl CoA, Carnitine. 


\section{CO-AUTHORSHIP}

This thesis was completed under the supervision and financial support of Dr. James Staples.

Contributions of co-authors:

JF Staples - Supervised study design and interpretation of results; provided comments and manuscript editing.

JCL Brown - Maintained the squirrel colony; assisted with liver mitochondrial isolation; provided manuscript editing.

MAC Cedrone - Assisted with Western blotting. 


\section{EPIGRAPH}

"Dude, sucking at something is the first step to becoming sorta good at something."

- Jake the dog, Adventure Time 


\section{ACKNOWLEDGEMENTS}

First I would like to acknowledge my supervisor, Dr. Jim Staples, for his patient guidance and support throughout this project. His leadership and great accessibility encouraged me beyond any setbacks I encountered. His genuine kindness and enthusiasm in and out of the lab have made this a wonderful experience for me. I could not have asked for a better supervisor.

Secondly I would like to acknowledge my advisory committee members: Dr. Louise Milligan and Dr. Denis Maxwell, for their valuable suggestions and criticism throughout the various, and sometimes challenging, stages of this project.

Next my lab mates: Dr. Jason Brown and Dillon Chung - they tirelessly answered all of my questions and provided endless (incredibly cynical) life advice. This has been the most significant academic challenge I have undertaken, and without their help I'm not sure it would have been possible.

I would like to express my great appreciation for the many people who generously provided their time to patiently walk me through various procedures and techniques. Lynda McCaig - for her dexterous and speedy dissections, and her unflagging cheerfulness on early mornings. Caitlin Vandermeer - for her meticulous explanation of enzyme assay protocol. I would like to express my extreme gratitude to Megan Cedrone who repeatedly donated time from a busy schedule to thoroughly train and assist me with all the molecular work related to this project. I would also like to offer special thanks to Lin Zhao for providing unrestricted access to his (seemingly) boundless knowledge of molecular techniques, and finally, Dr. Tim Regnault for access to his lab and equipment. 
I would also like to thank all the friends I have made here over the past two years. Dan Lim - between "gettin' huge" at the gym to realizing we are "dumb as rocks" when it comes to science, he has been an amazing friend through it all. Evelyn Boychuk - for being an excellent listener, cook, and co-consumer of fine hops-based-beverages. As well as all my office mates, past and present, who always made lunch-times an entertaining diversion.

Finally I would like to thank my family. My Mom and Mark - your unfaltering support and tireless employment as a sounding board have been vital in times of stress. My Dad and Gillian - between Sunday night dinners and family-personal-training days, you helped established an incredibly unstable cycle of binge-eating followed shortly after by regret and self-loathing at the gym. In any case both provided an outlet from the stress of grad-school, which was much needed and appreciated. My siblings: Nick, Taylor, Clay, and especially my little sister Jessica. She provided an island of home for me in London, and was not only eager to listen to all my problems, but to take my side with so much ferocity that I end up defending my opponents just to keep things fair.

Thank you. 


\section{TABLE OF CONTENTS}

ABSTRACT

CO-AUTHORSHIP iii

EPIGRAPH iv

ACKNOWLEDGEMNETS $\quad$ V

TABLE OF CONTENTS vi

LIST OF FIGURES viii

LIST OF APPENDICES $\quad \mathrm{x}$

LIST OF ABBREVIATIONS AND SYMBOLS Xii

CHAPTER 1: INTRODUCTION

$1.1 \quad$ Hibernation. 1

1.2 Mitochondria and cellular respiration. 5

$1.3 \quad$ Inhibition of succinate transport at the dicarboxylate 9 transporter.

$1.4 \quad$ Fatty-acyl CoAs, carnitine and carnitine palmitoyl $\quad 13$ transferase.

$\begin{array}{lll}1.5 & \text { Objectives and hypothesis. } & 17\end{array}$

CHAPTER 2: MATERIALS AND METHODS

$2.1 \quad$ Animal Capture and Care 18

$\begin{array}{lll}2.2 & \text { Radiotelemetry implants. } & 19\end{array}$

$\begin{array}{lll}2.3 & \text { Hibernation. } & 19\end{array}$

$2.4 \quad$ Liver mitochondria isolation. 20

$\begin{array}{lll}2.5 .1 & \text { Mitochondrial respiration. } & 21\end{array}$

$\begin{array}{lll}2.5 .2 & \text { Butylmalonate. } & 23\end{array}$

2.5.3 Palmitoyl CoA and Carnitine 23

$2.6 \quad$ Protein concentrations. 24

$2.7 \quad$ Carnitine-palmitoyl transferase activity assay. 26

$\begin{array}{lll}2.8 & \text { Protein quantification by Western blotting. }\end{array}$ 
$2.9 \quad$ Cytochrome $\mathrm{C}$ oxidase activity assay.

\section{CHAPTER 3: RESULTS}

3.1 Whole animal characteristics. 31

$3.2 \quad$ Mitochondrial respiration. 31

$3.3 \quad$ No difference in ETC complexes II-V subunit abundance 38 among metabolic states.

3.4 Suppression of mitochondrial respiration by

butylmalonate, palmitoyl CoA and the effect of carnitine.

3.5 Effect of carnitine on mitochondrial respiration. 40

3.6 Carnitine palmitoyl transferase activity among metabolic 45 states.

CHAPTER 4: DISCUSSION

4.1 Palmitoyl CoA and butylmalonate cause suppression of succinate-fuelled respiration rates.

4.2 Carnitine does not reverse respiratory suppression in either torpid or palmitoyl CoA treated mitochondria.

4.3 Liver mitochondrial respiration in torpid, interbout euthermic (IBE) and summer 13-lined ground squirrels. 


\section{LIST OF FIGURES}

Figure Title $\quad$ Page

1.1 Diagram of the electron transport chain (ETC).

1.2 Relationship between the dicarboxylate transporter (DCT), $\mathrm{P}_{\mathrm{i}^{-}} \quad 11$

$\mathrm{OH}^{-}$antiporter and the proton motive force $(\Delta \mathrm{P})$ generated by the electron transport chain (ETC).

1.3 Transport of fatty acyl CoAs into the mitochondrial matrix and $\quad 14$ subsequent $\beta$-oxidation.

1.4 Proposed effect of fatty acyl-CoAs and succinate transport on $\quad 16$ mitochondrial respiration.

2.1 The effects of palmitoyl CoA and carnitine on succinate-fuelled 25 mitochondrial respiration.

3.1 Mass of 13-lined ground squirrels (Ictidomys tridecemlineatus) 32 from May 2012-March 2013.

3.2 Core body temperature of a hibernating 13-lined ground squirrel (Ictidomys tridecemlineatus) measured by a temperature sensing radiotelemetry implant in the present study.

3.3 Succinate-fuelled State 3 and State 4 respiration rates of liver mitochondria of summer, interbout euthermic (IBE) and torpid 13 -lined ground squirrels measured in vitro at $37^{\circ} \mathrm{C}$.

3.4 Cytochrome $\mathrm{C}$ oxidase activity is significantly higher in torpid mitochondria than mitochondria from summer and IBE animals.

3.5 Succinate-fuelled State 3 and State 4 respiration rates relative to cytochrome c oxidase activity of liver mitochondria among metabolic states.

3.6 Protein abundance of electron transport chain (ETC) complexes $\mathrm{I}-\mathrm{V}$ subunits among metabolic states, analyzed by Western blot analysis.

3.7 Titration of inhibitory effect of palmitoyl CoA on state 3 succinate-fuelled respiration in 13-lined ground squirrel 
mitochondria.

3.8 Palmitoyl CoA suppresses succinate-fuelled state 3 respiration rates of liver mitochondria of torpid and interbout euthermic (IBE) 13-lined ground squirrels.

3.9 Percent suppression of respiration rate in interbout euthermic (IBE) and torpid liver mitochondria following treatment with butylmalonate, Palmitoyl CoA and Carnitine compared to paired control.

3.10 Carnitine has no rescuing effect on respiration rates of the liver mitochondria of torpid 13-lined ground squirrels.

3.11 Carnitine palmitoyl transferase activity among metabolic states. 46

4.1 Adjusted model of the proposed effect of fatty acyl-CoAs and 49 succinate transport on mitochondrial respiration. 


\section{LIST OF APPENDICIES}

Appendix Title $\quad$ Page

A.1 Animal Use Protocol (\#2012-016) 67

B.1 Palmitoyl-carnitine has no effect on state 3 succinate 68

fuelled mitochondrial respiration of liver mitochondria in interbout euthermic (IBE) and torpid 13-lined ground squirrels at $37^{\circ} \mathrm{C}$. 


\section{LIST OF ABBREVIATIONS AND SYMBOLS}

$\Delta \mathrm{A} \quad$ Change in absorbance $/ \mathrm{min}$

ADP Adenosine diphosphate

ANOVA Analysis of Variance

ANT Adenine nucleotide translocator

ATP Adenosine triphosphate

BCA Bicinchroninic acid

BMR Basal metabolic rate

BPM Beats per minute

BSA Bovine serum albumin

CAT carnitine-acylcarnitine translocase

CoQ Coenzyme Q

CPT I Carnitine palmitoyl transferase I

CPT II Carnitine palmitoyl transferase II

CytC Cytochrome C

D Dilution factor

DCT Dicarboxylate transporter

DTNB 5, 5'-Dithiobis-(2-Nitrobenzoic Acid)

$\varepsilon \quad$ Extinciton coefficient

EDTA Ethylene diamine tetraacetic acid

EGTA Ethylene glycol tetraacetic acid

EST Eastern standard time

ETC Electron transport chain 


\begin{tabular}{ll} 
HEPES & 4-(2-hydroxyethyl)-1-piperazineethanesulfonic acid \\
IBE & Interbout euthermia \\
IMM & Inner mitochondrial membrane \\
IMS & Intermembrane space \\
LCAD & Long-chain specific acyl CoA dehydrogenase \\
LSD & Least squares difference \\
MES & 2-(N-morpholino)ethanesulfonic acid \\
MiR05 & Mitochondrial respiration buffer No. 5 \\
MR & Metabolic rate \\
NADH & Nicotinamide adenine dinucleotide (reduced) \\
OMM & Outer mitochondrial membrane \\
$\triangle P$ & Proton motive force \\
$\mathrm{P}_{\mathrm{i}}$ & Inorganic phosphate \\
RCR & Respiratory control ratio \\
SDH & Succinate dehydrogenase \\
SDS & Sodium dodecyl sulfate \\
SEM & Standard error of mean \\
Tb & Core body temperature \\
TBST & Tris-Buffered Saline and Tween 20 \\
TRIS Base & Trisaminomethane Base \\
TRIS HCl & Trisaminomethane hydrochloride \\
V & Voltage dependent anion channel \\
\hline
\end{tabular}




\section{CHAPTER 1 \\ INTRODUCTION}

\subsection{Hibernation}

Due to planetary rotation and tilt, ambient temperatures across the world are by no means constant, and seasonal fluctuations are an inescapable challenge for life on Earth. Biochemical reaction rates are directly proportional to temperature, including the enzymatic reactions occurring within the bodies of animals (Hochachka and Somero, 2002). For poikilothermic ectotherms, organisms with low metabolic rate (MR) that allow their internal body temperature $\left(\mathrm{T}_{\mathrm{b}}\right)$ to vary with ambient temperature, the rate of internal reactions is acutely vulnerable to fluctuations in ambient temperature (Hill et al., 2008). As a result, ectotherms are typically limited by behavioural thermoregulation to maintain a preferable body temperature, limiting their distribution and activity in cooler environments (Hill et al., 2008). Alternatively, homeothermic endotherms actively maintain a fairly constant $T_{b}$, independent from ambient temperature using internally generated heat (Bennett and Ruben, 1979). Although this endothermic strategy requires a considerably higher MR, its presumed advantage is that rates of enzymatic activity do not necessarily depend on ambient temperature, and function reliably within a particular temperature range, enabling sustained activity independent of fluctuations in habitat temperature (Hochachka and Somero, 2002). However, endothermy is energetically costly; a typical endotherm needs to consume as much as 10-fold more calories than a similarly sized ectotherm (White et al., 2006). While ectotherms can go for long periods without eating, typical endotherms need to eat frequently to meet their high caloric requirements. 
When ambient temperature drops below an endotherm's thermal neutral zone (the range of ambient temperature an endotherm can tolerate without increasing MR to maintain $T_{b}$ ), the requirement for food becomes particularly imperative, as endotherms must increase their metabolically produced heat in proportion to the drop in ambient temperature in order to maintain a constant $\mathrm{T}_{\mathrm{b}}$ (Hill et al., 2008). The smaller an endothermic mammal is, the higher the surface area to volume ratio, and the greater the heat loss to the environment (Geiser, 2004; Hochachka and Somero, 2002). Therefore small endotherms living in temperate and polar climates lose a large portion of their metabolically generated heat to the environment, which in turn causes a large caloric requirement to maintain a constant $\mathrm{T}_{\mathrm{b}}$ (Geiser, 2004). This requirement is compounded during the coldest seasons, when metabolic demand is at its peak, and food availability is typically at its lowest. Endotherms living in seasonally cold climates have evolved various strategies to successfully cope with the energetic stress of winter, such as building insulated nests (Casey, 1981), increasing both fur (Hart, 1956) and fat insulation (Young, 1976), and caching food (Smith and Reichman, 1984). Other strategies aim to avoid the stress altogether, such as migrating to warmer, calorie-rich environments (Hill et al., 2008). An alternate approach to seasonal survival is to reduce energy demands by decreasing $\mathrm{MR}$ and, consequently $\mathrm{T}_{\mathrm{b}}$, in a process known as hibernation (Reviewed in: Staples and Brown, 2008).

Hibernation, although not an overly common strategy, is employed by a diverse range of mammalian species. Representatives appear in as many as eight orders, including monotremes, marsupials, rodents, primates, bats (Geiser, 2004), and the oftencontested large carnivore, the American black bear, that exhibit similar, but limited 
characteristics of true hibernators (Tøien et al., 2011). There is even one species of bird, Phalaenoptilus nuttallii, that is a documented hibernator (Geiser, 2004). This curious distribution across many distantly related taxa suggests that the ability to hibernate was perhaps a common ancestral trait, and is conserved within the genomes of all mammals, but only expressed in a small few (Srere et al., 1992). One of the most widely studied of these hibernating species is the 13-lined ground squirrel (Rodentia: Sciuridae, Ictydomys tridecemlineatus), the species used in all work related to this thesis.

For the 13-lined ground squirrel, warm spring weather signals the beginning of a brief period of reproduction, followed by intense foraging throughout the summer in order to develop massive endogenous fat stores, which will supply all the energy required for the upcoming hibernation season. Work in our own lab has observed pre-hibernation body masses in October to be $55 \%$ greater than post-hibernation body masses recorded in March (figure 3.1), which is similar to increases observed in other hibernating species (Armitage et al., 2013; Mckeever, 1963). Typical of most hibernators, once hibernation begins, 13-lined ground squirrels do not eat or drink at all, and the digestive tract degenerates for the 5-7 months of their hibernation season (Carey et al., 2003).

In late fall ground squirrels enter the underground burrow where they spend the entire hibernation season. Hibernation is characterized by lengthy bouts of metabolic suppression known as torpor, (lasting from 5-14 days), interrupted by brief periods ( $~ 8$ hours) of increased metabolic activity known as interbout euthermia (IBE) (Figure 3.2). In this way hibernators are able to conserve $\sim 90 \%$ of the energy expended by their homeothermic counterparts (Wang, 1979). During torpor, metabolic rate (MR, approximated by whole animal oxygen consumption) is reduced to $\sim 5 \%$ of basal 
metabolic rate (BMR; Geiser, 2004), and $\mathrm{T}_{\mathrm{b}}$ subsequently falls, and is maintained a few degrees above ambient temperature. Despite the cyclical rise and fall of $T_{b}$ seen between torpor and IBE, hypothalamic regulation of $\mathrm{T}_{\mathrm{b}}$ remains functional throughout the hibernation season. For example, in two closely related species, golden mantled ground squirrels and yellow bellied marmots, if ambient temperature in the burrow drops below $5^{\circ} \mathrm{C}$ the hypothalamus stimulates an increase in metabolism to defend a thermoregulatory set-point of $5^{\circ} \mathrm{C}$ during torpor (Florant and Heller, 1977; Heller and Hammel, 1972). During periods of torpor in 13-lined ground squirrels suppression of $M R$ and $T_{b}$ are paralleled by decreases in ventilation (from 100-200 to 1-3 breaths per minute) and heart rate (from $\sim 300 \mathrm{bpm}$ to as 3-15bpm) (Landau and Dawe, 1958). Arousal from a torpor bout occurs spontaneously: MR rapidly increases and $T_{b}$ is returned to euthermic levels $\left(\sim 37^{\circ} \mathrm{C}\right)$ via shivering and non-shivering thermogenesis (reviewed in Staples and Brown, 2008). These brief periods of intense metabolic activity consume $\sim 80 \%$ of the energy used throughout the hibernation season (Heldmaier et al., 1993; Wang, 1978; Wang, 1979), however the reason they occur remains unknown. Spontaneous arousals have been suggested to be necessary for proper immune function (Prendergast et al., 2002), or a requirement for adequate sleep (Daan et al., 1991). Regardless of the physiological reason for their occurrence, transitions between IBE and torpor are remarkable because not only do hibernators undertake an extreme modification to $M R$ and $T_{b}$, but they do so rapidly (within hours), and repeatedly.

The suppression of MR as a hibernator concludes a period of IBE and enters a bout of torpor is known as 'entrance', and involves active inhibition of metabolism in addition to passive thermal effects due to decreasing $T_{b}$ (Geiser, 2004; Staples and Brown, 2008). 
In a hibernator entering a bout of torpor suppression of MR precedes any decrease in $T_{b}$ (Chung et al., 2011; Heldmaier et al., 2004). So although the reduction in $\mathrm{T}_{\mathrm{b}}$ will depress biochemical reaction rates, it is active suppression of metabolism that initiates and maintains very low MR in torpor. In fact it has been estimated that active suppression accounts for as much as $40-70 \%$ of the decrease in MR observed in hibernation (reviewed in Staples and Brown, 2008). Although there have been many attempts to elucidate and understand the suppression of MR observed during torpor, the mechanisms driving suppression remains unclear.

\subsection{Mitochondria and cellular respiration.}

Mitochondria are the greatest contributor to whole animal MR; being responsible for $\sim 90 \%$ of whole-animal oxygen consumption in mammals (Rolfe and Brown, 1997), as well as being the primary site of adenosine triphosphate (ATP) synthesis in the cell. An overwhelming number of processes are driven by ATP-consumption, such as protein synthesis, $\mathrm{Na}^{+} / \mathrm{K}^{+}$and $\mathrm{Ca}^{2+}$-ATPase pumps and gluconeogenesis to name a few. Altogether it is estimated that $70-80 \%$ of the mammalian BMR can be contributed to ATP-consuming processes (Rolfe and Brown, 1997), which means that any depression in ATP production has the potential to disturb all of the above processes. Thus when examining suppression of MR as seen in hibernation, mitochondria are the primary site of investigation.

Mitochondria are bounded by a freely permeable outer mitochondrial membrane (OMM) whose sieve-like properties have been attributed to the abundance of voltage dependent anion-selective channels (VDAC), that facilitate passage of most molecules

smaller than 1.5kDa (Bernardi et al., 2006; Rostovtseva and Bezrukov, 2008). By contrast 
the inner mitochondrial membrane (IMM) is effectively impermeable, but has many transporters to facilitate selective passage into and out of the mitochondrial matrix. It is the relative impermeability of the IMM that allows for the generation of the proton motive force $(\Delta \mathrm{P})$ which powers ATP synthesis. Within the mitochondrial matrix, Krebs cycle products NADH and succinate are oxidised by IMM-bound electron transport chain (ETC) complexes I and II respectively. Electrons are then passed down a chain of progressively more electronegative acceptors, at each exchange releasing free energy, until reaching the terminal electron acceptor oxygen $\left(\mathrm{O}_{2}\right)$, at ETC complex IV. Some of the free energy released at each exchange of electrons is used by complexes I, III, and IV to actively pump protons $\left(\mathrm{H}^{+}\right)$across the IMM into the inter membrane space (IMS). This process establishes $\Delta \mathrm{P}$, the electrochemical gradient that is used by ETC complex V $\left(\mathrm{F}_{1} \mathrm{~F}_{\mathrm{O}}\right.$ ATP synthase) to drive ATP synthesis from adenosine diphosphate (ADP) and inorganic phosphate $\left(\mathrm{P}_{\mathrm{i}}\right.$; figure 1.1).

Several investigators have compared the metabolism of mitochondria isolated from both torpid, and euthermic (IBE and/or summer) hibernators to determine the extent of active inhibition of metabolism. Numerous studies have reported that succinate-fuelled respiration is up to $70 \%$ lower compared with euthermic controls, when assayed at the same in vitro temperature $\left(37^{\circ} \mathrm{C}\right.$; Armstrong and Staples, 2010; Barger et al., 2003; Brown et al., 2012; Chung et al., 2011; Muleme et al., 2006; Pehowich and Wang, 1984). The liver is an excellent tissue to study because it is large, easy to dissect and protocols for mitochondrial isolation are well established. Moreover liver is metabolically significant to mammals because, despite contributing only $\sim 5 \%$ of total body mass, it consumes $\sim 20 \%$ of total inspired oxygen at rest (in rats; Rolfe and Brown, 1997). For 


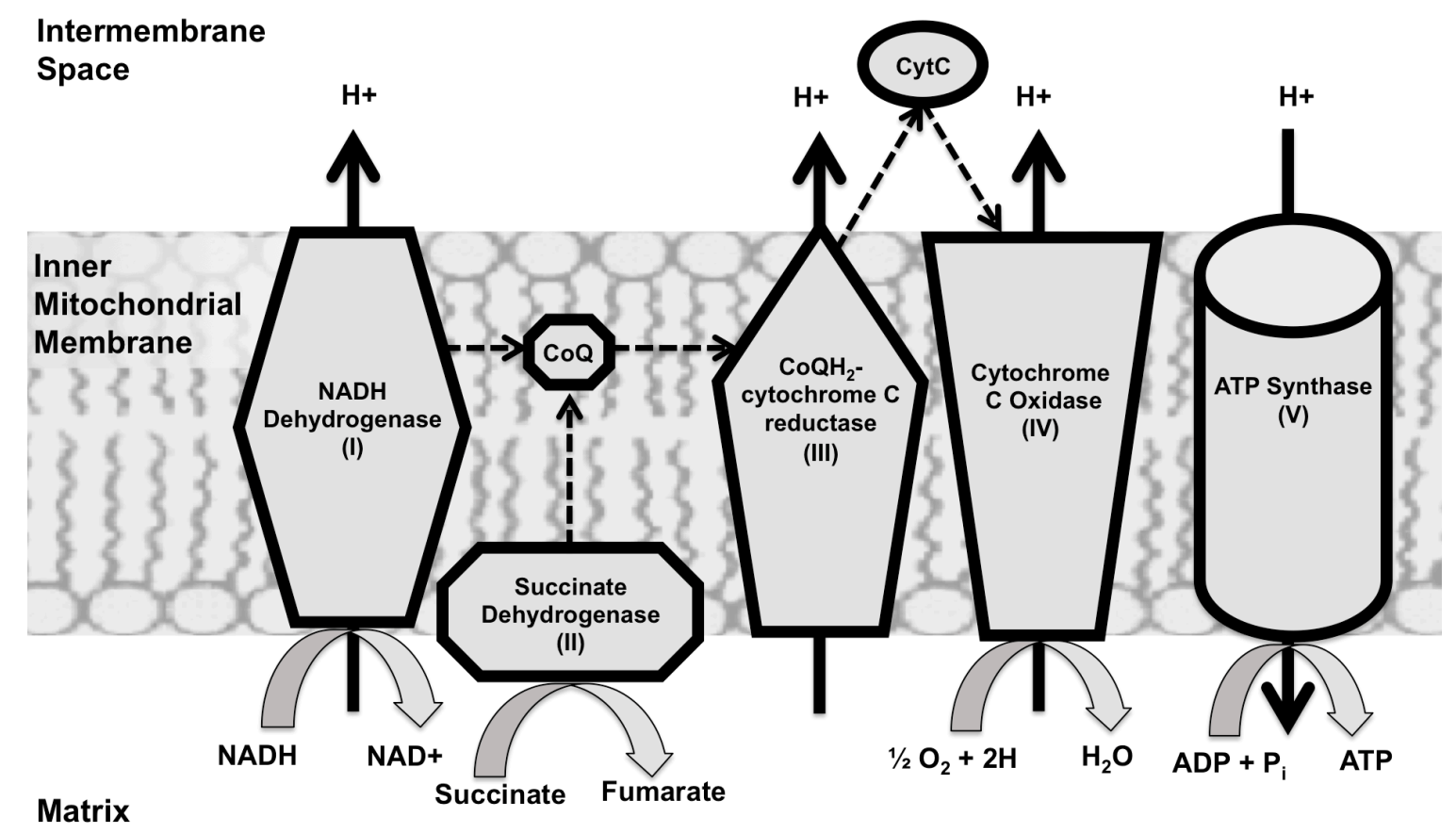

Figure 1.1 Diagram of the electron transport chain (ETC). Succinate and NADH are oxidised at complexes II and I respectively, surrendering electrons that then travel down a chain of increasingly electronegative acceptors (in order: Coenzyme Q (CoQ), Complex III, Cytochome C (CytC), Complex IV; path is shown as dashed arrows). The passing of electrons from complex to complex releases free energy that is then used to actively pump hydrogen ions $\left(\mathrm{H}^{+}\right)$across the inner mitochondrial membrane (IMM) into the intermembrane space (IMS) at complexes I, III and IV (solid black arrows), ultimately establishing an electrochemical gradient known as the proton motive force $(\Delta \mathrm{P})$. Complex IV (ATP synthase) employs the $\triangle \mathrm{P}$ to drive the synthesis of ATP from ADP and inorganic phosphate $\left(\mathrm{P}_{\mathrm{i}}\right)$ as hydrogen ions flow back across the inner mitochondrial membrane into the matrix. Electrons flowing down the ETC are ultimately donated to the terminal electron acceptor: oxygen $\left(\mathrm{O}_{2}\right)$, at complex IV. Figure is based on a review by Hatiefi (1985). 
comparison, skeletal muscle contributes $\sim 42 \%$ of total body mass, and only consumes $\sim 30 \%$ of total inspired oxygen (in rats; Rolfe and Brown, 1997), however succinatefuelled respiration in skeletal muscle is merely $\sim 30 \%$ suppressed in torpor relative to euthermia $\left(37^{\circ} \mathrm{C}\right.$; Brown et al., 2012).

Muleme et al. (2006) attempted to clarify where mitochondrial metabolic suppression was occurring in torpor by measuring respiration rates through portions of the ETC that were functionally isolated using selective inhibitors. They determined that in torpor, respiration was inhibited between succinate dehydrogenase (SDH; ETC complex II) and cytochrome $c$ oxidase (complex IV), and that this suppression was then reversed in IBE animals (Muleme et al., 2006). Subsequently Armstrong and Staples (2010) as well as Chung et. al (2011) found significant inhibition of SDH by oxaloacetate in torpor which was reversed by pre-incubation with isocitrate. However, despite this reversal, torpid respiration still remained four-fold lower than euthermic rates, indicating that inhibition of SDH by oxaloacetate does not account for the majority of suppression seen in torpor. Additionally, Brown et al. (2013) found that while SDH activity was suppressed in torpor at $37^{\circ} \mathrm{C}$, it was not suppressed when measured at $10^{\circ} \mathrm{C}$, suggesting that SDH may contribute in the initiation of suppression, but does not maintain it. Finally, in both liver and skeletal muscle, torpid mitochondria have a greater affinity for succinate than euthermic mitochondria (Brown et al., 2013). Which suggests that decreased affinity is not the underlying cause of respiratory suppression in torpor (Brown et al., 2013).

The pattern of mitochondrial metabolic suppression differs fundamentally depending on whether the hibernating animal is the process of suppression during entrance, or reversal of that suppression during arousal. During entrance, when an animal goes from 
euthermia $\left(\mathrm{T}_{\mathrm{b}}=37^{\circ} \mathrm{C}\right)$ into torpor, the suppression of succinate-fuelled respiration rate occurs rapidly, and full suppression ( $70 \%)$ is achieved within the first 2 hours it takes $\mathrm{T}_{\mathrm{b}}$ to fall to $30^{\circ} \mathrm{C}$ (Chung et al., 2011). Conversely when a torpid $\left(\mathrm{T}_{\mathrm{b}}=5^{\circ} \mathrm{C}\right)$ animal spontaneously arouses, suppression of succinate-fuelled mitochondrial respiration is reversed much more slowly, with respiration rate still $\sim 50 \%$ lower than euthermic level by the time $\mathrm{T}_{\mathrm{b}}$ has increased from $5^{\circ} \mathrm{C}$ to $30^{\circ} \mathrm{C}$ (Armstrong and Staples, 2010). Taken together the rapid suppression of respiration at relatively high $T_{b}$ during entrance, and the gradual reversal of suppression when $\mathrm{T}_{\mathrm{b}}$ starts at $5^{\circ} \mathrm{C}$ during arousal (termed "fast-in, slow-out") suggests that the mechanism behind suppression is, in some way, temperature dependent. Furthermore the distribution of hibernators across distantly related taxa suggests that hibernation is a phenotype that either arose or was lost repeatedly, implying a simple reprogramming of existing mammalian genes. Collectively, the above work provides a framework for criteria that a potential mechanism governing suppression in hibernation must meet; a mechanism must be fast (hours) and readily reversible, it must occur between ETC complex II and IV, and is likely temperature-sensitive. Inhibition of succinate transport into the mitochondrial matrix has the potential to meet these criteria, and is the possible mechanism of suppression I chose to investigate.

\subsection{Inhibition of succinate transport at the dicarboxylate transporter.}

Succinate freely diffuses across the OMM, facilitated by VDAC, but in order to cross the IMM succinate must be transported into the mitochondrial matrix by the dicarboxylate transporter (DCT). The DCT is located in the IMM, and catalyzes the exchange of the dicarboxylate anions succinate and/or malate for $\mathrm{P}_{\mathrm{i}}$ (Johnson and Chappell, 1973). The inorganic sulphur-containing compounds; sulphate, sulfite and 
thiosulfate, are also transported by the DCT (Crompton et al., 1974). The DCT has two separate binding sites; one for phosphate and thiosulphate, and the other for dicarboxylates, sulphate and sulfite, which together operate by a sequential antiport mechanism (Indiveri et al., 1993). The DCT is thought to work in tandem with the $\mathrm{P}_{\mathrm{i}}-\mathrm{OH}^{-}$ antiporter, whereby $\mathrm{P}_{\mathrm{i}}$ enters the mitochondrial matrix in exchange for $\mathrm{OH}^{-}$, an exchange driven by the $\mathrm{pH}$ gradient of the $\Delta \mathrm{P}$ (McGivan and Klingenberg, 1971). The resulting phosphate gradient acts to catalyze the action of the DCT, moving dicarboxylates into the matrix and ultimately recycling $\mathrm{P}_{\mathrm{i}}$ back out of the matrix, all at the expense of $\Delta \mathrm{P}$ (Coty and Pendersen, 1975, figure 1.2). The DCT can be inhibited by IMM impermeable dicarboxylate analogues, the most commonly used being butylmalonate (Johnson and Chappell, 1973; Robinson and Chappell, 1967; Robinson and Williams, 1970). Inhibition of the DCT precludes succinate transport into the mitochondrial matrix, and consequently succinate-fuelled respiration is suppressed; a result similar to that observed in torpid hibernators.

It has been repeatedly reported that long chain acyl co-enzyme A conjugates (hereafter referred to as long chain acyl CoAs) inhibit a variety of IMM transport systems, including the DCT (Ventura et al., 2005), resulting in suppression of mitochondrial respiration (Pande and Blanchaer, 1971; Shrago et al., 1995; Shug et al., 1971; Wojtczak and Zaluska, 1967; Woldegiorgis et al., 1982). The ability for fatty acyl CoAs to act as competitive inhibitors is likely due to their negatively charged adenosinephosphate head group, which is suggested to bind and block the active site of a variety of transporters and enzymes, while the long lipophilic tail anchors the acyl-CoA to the 


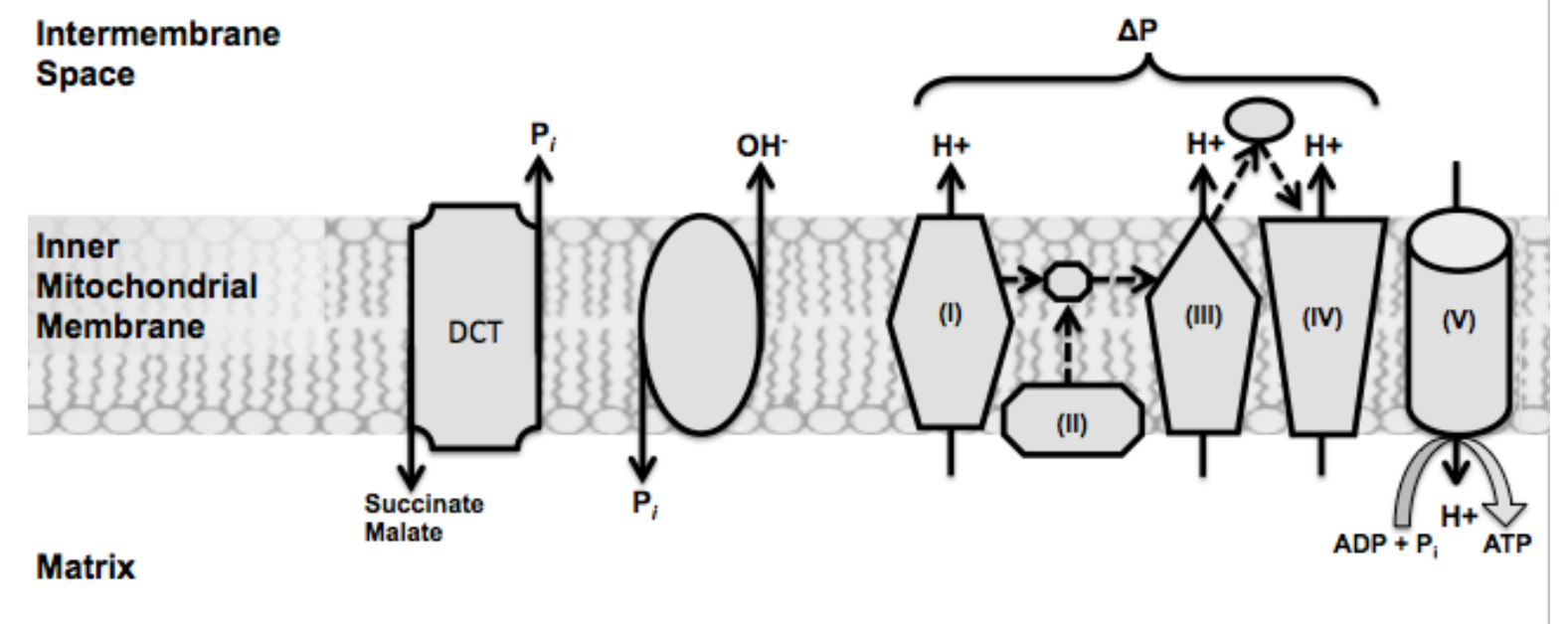

Figure 1.2 Relationship between the dicarboxylate transporter (DCT), $\mathrm{P}_{\mathrm{i}}-\mathrm{OH}^{-}$ antiporter and the proton motive force $(\Delta P)$ generated by the electron transport chain (ETC). The $\triangle \mathrm{P}$ generated by the ETC establishes a $\mathrm{pH}$-gradient that then acts to drive the exchange of $\mathrm{P}_{\mathrm{i}}$ for $\mathrm{OH}^{-}$at the $\mathrm{P}_{\mathrm{i}} \mathrm{OH}^{-}$antiporter. This in turn establishes a $\mathrm{P}_{\mathrm{i}}$ gradient that catalyzes the sequential antiport mechanism that drives exchange of dicarboxylates (succinate and malate) for $\mathrm{P}_{\mathrm{i}}$ by the DCT. Figure based on models by McGivan and Klingenberg (1971), and Coty and Pendersen (1975). 
membrane phospholipid domain, maintaining the head group in close proximity to these transporters (Duszyinski and Wojtczak, 1974; Shrago et al., 1995; Wojtczak, 1976). This anchoring mechanism could act to keep fatty acyl CoAs associated with the mitochondrial membrane, and in position to inhibit the DCT, even following the mechanical disruption associated with tissue homogenization and subsequent isolation of mitochondria, possibly explaining why suppression is able to persist in in vitro assays of mitochondrial respiration in studies of hibernators. Additionally, Chung et al. (2013) reported changes in the phosphorylation state of long-chain specific acyl CoA dehydrogenase (LCAD, responsible for catalyzing $\beta$-oxidation of fatty acyl CoAs) between torpid and summer animals. The ability to reversibly phosphorylate LCAD suggests that hibernators can regulate the rate of fatty acyl CoA metabolism, further implicating fatty acyl CoAs as a potential mechanism behind the reversible suppression of mitochondrial respiration in hibernation.

As previously discussed, hibernators subsist on endogenous fat stores throughout the hibernation season (Carey et al., 2003), and this is confirmed by a respiratory quotient of $\sim 0.70$, which indicates exclusively lipid metabolism (Buck and Barnes, 2000). It is therefore not surprising that the level of free fatty acids in the plasma of hibernators is elevated for the duration of the hibernation season (Florant et al., 1990). From the plasma, free fatty acids diffuse into tissues and are converted into fatty acyl CoAs in the cytosol before being metabolized by beta-oxidation. The extensive use of fats by hibernators supports the hypothesis that metabolic regulation by fatty acyl CoAs is a potentially viable mechanism underlying the suppression of succinate-fuelled respiration in torpid mitochondria. 


\subsection{Fatty-acyl CoAs, carnitine and carnitine palmitoyl transferase.}

In the cytosol, long chain fatty acids are activated by an enzyme embedded in the OMM called long chain acyl CoA synthetase, forming fatty acyl CoAs (Kerner and Hoppel, 2000). Fatty acyl CoAs then diffuse into the IMS via VDAC (Kerner and Hoppel, 2000). From there fatty acyl CoAs are transported into the matrix in a two step process (reviewed in Kerner and Hoppel, 2000; McGarry and Brown, 1997). First, the enzyme carnitine palmitoyl transferase I (CPT I, located on the OMM) catalyzes the conjugation of the fatty acyl CoA with carnitine, forming acyl-carnitine. Next, acylcarnitne is transported across the IMM by carnitine-acylcarnitine translocase (CAT). On the matrix side of the IMM carnitine palmitoyl transferase II (CPT II) catalyzes the release of carnitine from the fatty acyl CoA, and the released carnitine is recycled back across the IMM by CAT (Pande, 1975). The fatty acyl CoA, now in the mitochondrial matrix, undergoes $\beta$-oxidation to acetyl CoA, which then enters the Krebs cycle, whose products ultimately fuel the ETC and mitochondrial respiration (for summary see figure $1.3)$.

Carnitine is necessary for fatty acyl CoA transport into the matrix by CAT (Fritz and Marquis, 1965). It follows, therefore, that, in the absence of carnitine, fatty acyl CoA transport into the matrix would cease, and all downstream processes of lipid metabolism ( $\beta$-oxidation, oxidation at the Krebs cycle, substrates fuelling the ETC) would halt. It has been suggested that in the absence of carnitine, fatty acyl CoAs would then accumulate in the IMS, and as a result inhibit mitochondrial transporters, including DCT, ultimately causing suppression any mitochondrial respiration that depends on substrates transported by the DCT (Pande and Blanchaer, 1971; Ventura et al., 1995; Ventura et al., 2005). 


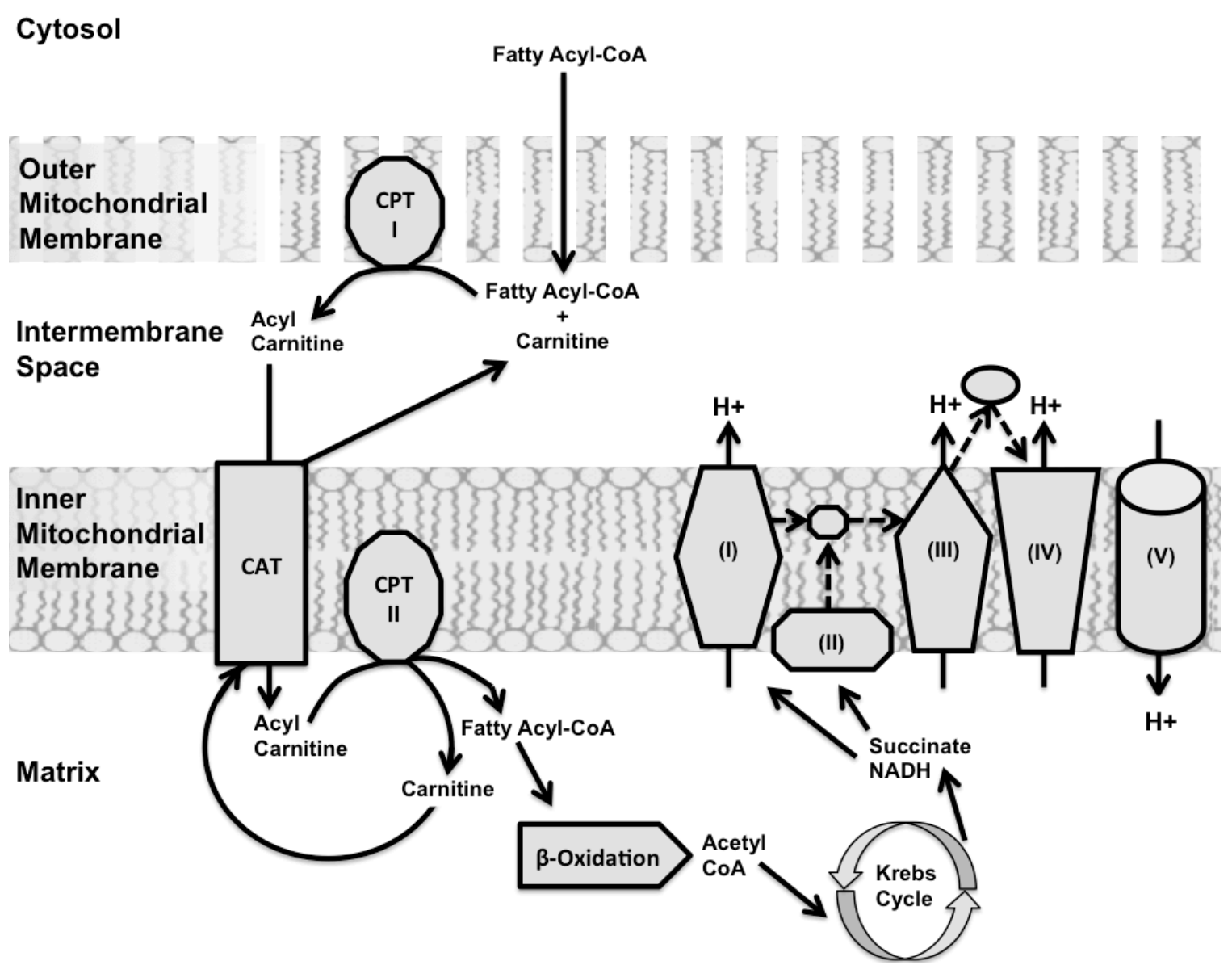

Figure 1.3 Transport of fatty acyl CoAs into the mitochondrial matrix and subsequent $\boldsymbol{\beta}$-oxidation. Fatty acyl CoAs from the cytosol diffuse across the outer mitochondrial membrane (OMM), and are conjugated with carnitine by carnitine palmitoyl transferase I (CPT I). Carnitine-acylcarnitine transferase (CAT) then transports acyl carnitine into the mitochondrial matrix where the fatty acyl CoA is cleaved from carnitine by carnitine palmitoyl transferase II (CPT II). Carnitine is transported out of the matrix by CAT, and the fatty acyl CoA undergoes $\beta$-oxidation to acetyl-CoA. Acetyl CoA enters the Krebs cycle, which produces substrates for ETC oxidation, ultimately fuelling mitochondrial respiration. Figure is based on the work of Kerner and Hoppel (2000), McGarry and Brown (1997), and Pande (1975). 
Further building on the idea that suppression in hibernators is due to an accumulation of fatty acyl CoAs in the absence of carnitine, is work on carnitine metabolomics by Nelson et al. (2009), who found higher levels of carnitine in the livers of euthermic animals (IBE and summer), compared with torpid animals. This supports the idea that fatty acyl CoAs accumulate during torpor, inhibit mitochondrial transporters (such as the DCT), and are then removed by carnitine in periods of euthermia (figure 1.4).

In rat liver mitochondria treated with 2, 4 dinitrophenol (which uncouples substrate oxidation from ATP synthesis), Ventura et al. (2005) found that palmitoyl CoA (a 16C fatty acyl CoA) inhibited respiration powered by succinate oxidation, but not that powered by glutamate oxidation. As their experiments were performed in the absence of carnitine, they concluded that palmitoyl CoA inhibits succinate transport across the inner mitochondrial membrane at the DCT, while glutamate was able to enter the inner mitochondrial membrane uninhibited via the $\mathrm{H}^{+}$/glutamate carrier and aspartate glutamate carrier (Palmieri, 2004). Swelling studies confirmed that inhibition by palmitoyl CoA was occurring at the DCT (Ventura et al., 2005). In these studies mitochondria were placed in an isoosmotic suspension of ammonium succinate. Protonated succinate is exchanged for phosphate across the IMM by the DCT, meanwhile ammonia passively enters the matrix and binds with protons as they dissociate from the transported succinate, forming $\mathrm{NH}_{4}+$. This maintains $\mathrm{pH}$ balance, permitting continued succinate transport, but ultimately causes swelling by 'trapping' protons in the matrix, which is then measured as a change in absorbance over time due to decreased light scattering (LaNoue and Schoolwerth, 1979; Ventura et al., 2005). In the presence of the palmitoyl CoA Ventura 


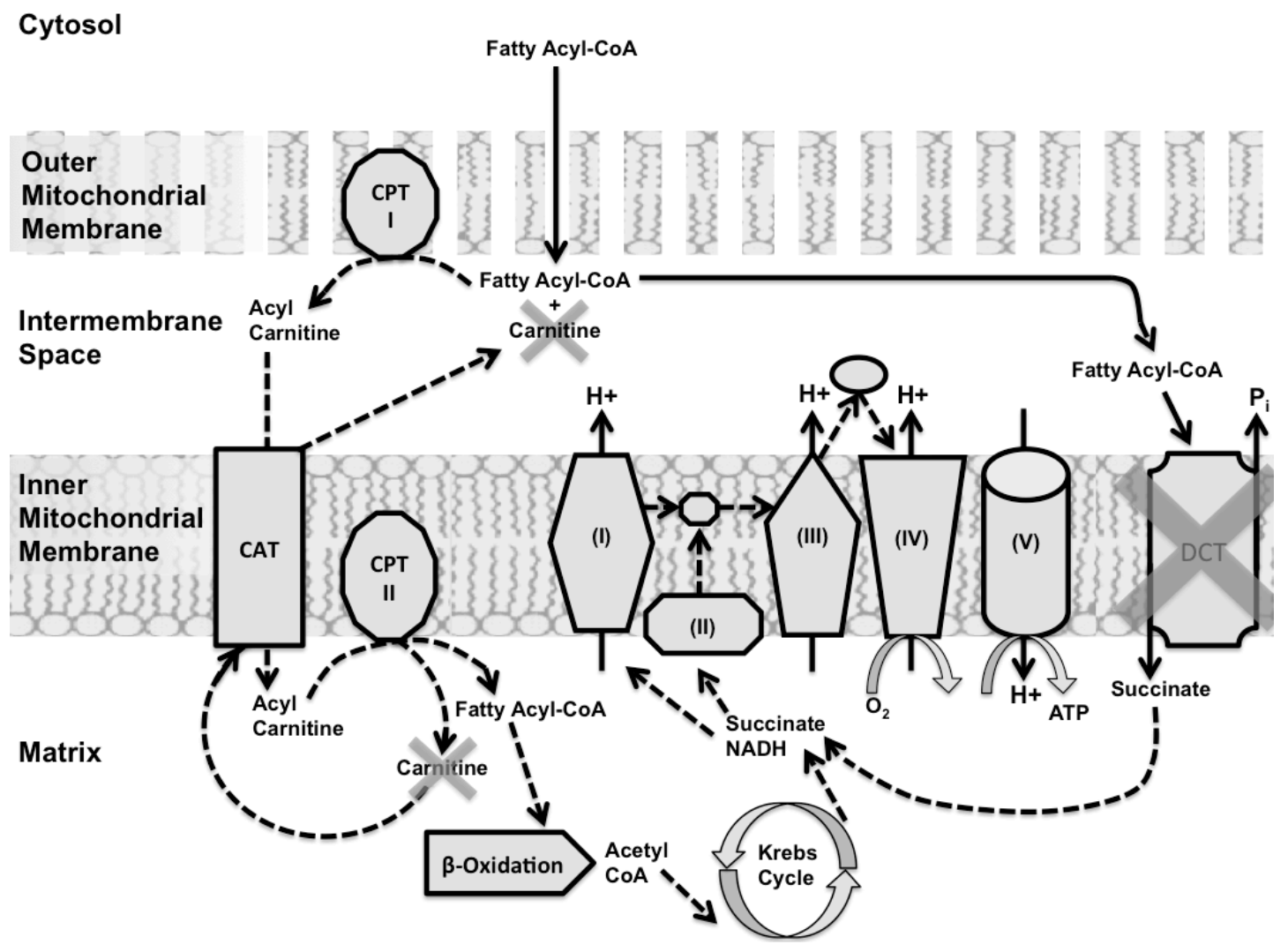

Figure 1.4 Proposed effect of fatty acyl-CoAs and succinate transport on

mitochondrial respiration. In the absence of carnitine the transport of fatty acyl-CoAs across the inner mitochondrial membrane (IMM) is halted. This leads to an accumulation of fatty acyl-CoAs in the inter-membrane space (IMS), which cause inhibition of the dicarboxylate transporter (DCT), preventing succinate transport into the matrix. Together this leads to the suppression of mitochondrial respiration. Solid lines indicate operating processes, dashed lines indicate inhibited processes. Figure is based on the proposed models of Ventura et al. $(1995,2005)$ and Pande and Blanchaer (1971). 
et al. (2005) found that swelling was significantly reduced relative to the control, indicating inhibition of succinate transport at the DCT by palmitoyl CoA.

In hibernators, Muleme et al. (2006) and Brown et al. (2012) showed mitochondrial respiratory suppression during torpor with succinate but not glutamate, indicating a possible role for inhibition of the DCT during torpor. Taken together there is compelling evidence that the suppression of succinate-fuelled respiration observed in torpid hibernators could reflect a disruption of succinate transport at the DCT, which could potentially be mediated by fatty acyl CoAs, and the availability of carnitine.

\subsection{Objectives and Hypothesis.}

The goal of this project is to identify a contributing mechanism responsible for the suppression of succinate-fuelled mitochondrial respiration rate in torpid mitochondria. I hypothesize that a mechanism behind the suppression of mitochondrial respiration during torpor is inhibition of succinate import across the IMM at the DCT by fatty acyl CoAs. I predict the suppression in liver mitochondrial succinate oxidation of torpid hibernators is a result of the inhibition of the DCT by long chain fatty acyl-CoAs. It is expected that the activity of the DCT will be significantly lower in torpid mitochondria than in euthermic mitochondria. Secondly, I predict that the addition of palmitoyl CoA to purified mitochondria will suppress the respiration of euthermic, but not torpid, mitochondria. Finally I predict that supplying carnitine will increase the respiration of torpid mitochondria by reversing acyl-CoA dependent inhibition of respiration, while it will have no effect on euthermic mitochondria. 


\section{CHAPTER 2}

\section{MATERIALS AND METHODS}

\subsection{Animal Capture and Care.}

All procedures were approved by the University of Western Ontario Animal Use Subcommittee (Protocol \#2012-016; Appendix A). Wild thirteen-lined ground squirrels (Ictydomys tridecemlineatus) were captured in Carman, MB, Canada (49³0’ $\mathrm{N}$, $98^{\circ} 01^{\prime} \mathrm{W}$ ) in May 2011 using live-traps. Additionally, any pups born to wild-caught females after capture were cared for and weaned following a protocol modified from Vaughan et al. (2006) and added to the pool of captive animals.

Following capture, animals were housed at $22 \pm 3^{\circ} \mathrm{C}$, with photoperiod adjusted weekly to match that of Carman, MB. Each animal was housed individually in a plastic shoebox cage $(26.7 \mathrm{~cm} \times 48.3 \mathrm{~cm} \times 20.3 \mathrm{~cm})$ on dried corn-cob bedding (Ren's Feed and Supply Ltd., Oakville, ON), with crinkled paper strips for nest building (EnviroDri, Shepherd, Milford, NJ) and a transparent red plastic tube for enrichment (Bio-Serv, Frenchtown, NJ). Animals were provided water and food (LabDiet 5P00, Lab Diet, St Louis, MO; Dog food, Iams) ad libitum, as well as a flavoured wooden chewing stick to help control incisor growth. Additionally, animals were supplemented with sunflower seeds ( $\sim 10$ seeds) every other day. Squirrels were visually inspected each day and were weighed weekly during cage cleaning (figure 3.1).

Wild caught animals were quarantined for a minimum of 42 days. During quarantine animals received biweekly subcutaneous injections of the cestocide Droncit 
$\left(5.68 \mathrm{mg} \cdot \mathrm{mL}^{-1}, 0.2 \mathrm{~mL} \cdot 250 \mathrm{~g}^{-1}\right.$; BayerDVM, Pittsburgh, PA). Fecal matter was

collected from each animal twice weekly, and sent to UWO Animal Care and Veterinary Services, where it was tested for endoparasites using Fecalyzer kits (Vétoquinol, Buena NJ). When two consecutive negative tests were returned, the animal was removed from quarantine.

\subsection{Radiotelemetry implants.}

Body temperature was measured using radiotelemetry. In July and August, animals were anesthetized using isoflourane gas $\left(4 \%\right.$, balance $\left.\mathrm{O}_{2}\right)$ and surgically implanted with intraperitoneal radio telemeter transmitters (TA-F20 or TA-F10, Data Sciences International, Arden Hills, MN) that regularly monitor and report each individual's $\mathrm{T}_{\mathrm{b}}$. Each animal was provided postoperative analgesia (a subcutaneous injection of ketoprofen; $\left.10 \mathrm{mg} \cdot \mathrm{mL}^{-1}, 0.05 \mathrm{~mL} \cdot 100 \mathrm{~g}^{-1}\right) . \mathrm{T}_{\mathrm{b}}$ was collected and recorded every 4 minutes using telemetry receivers (model RA1010 or RPC-1, Data Sciences International), and data acquisition software (Dataquest ART, Data Sciences International).

\subsection{Hibernation.}

In October, when bouts of torpor were observed (even while still housed at $22 \pm 3^{\circ} \mathrm{C}$ ) and animal weights had plateaued (figure 3.1), animals were transferred to environmental chambers where temperature was lowered $1{ }^{\circ} \mathrm{C}$ per day until it reached $4 \pm 2^{\circ} \mathrm{C}$. At this point, photoperiod was adjusted to $2 \mathrm{~h}$ light (8am-10am): $22 \mathrm{~h}$ dark. By early November all individuals were displaying torpor bouts, and after torpor was observed for one week, food (but not water) was withdrawn. In April torpor bouts spontaneously began to shorten, and animals were moved back into an animal care room, 
feeding was resumed, temperature returned to $22 \pm 3^{\circ} \mathrm{C}$, and photoperiod was restored to imitate that of Carman, MB.

\subsection{Liver mitochondria isolation.}

Experimental groups were comprised of summer, torpid, and IBE animals. Summer animals were sampled throughout July and August at 8:30am $\pm 30 \mathrm{~min}$ EST, $\mathrm{T}_{\mathrm{b}}=$ $37 \pm 1^{\circ} \mathrm{C}$. Torpid and IBE animals were sampled throughout January and February. Animals were considered torpid when $\mathrm{T}_{\mathrm{b}}$ was $5 \pm 1{ }^{\circ} \mathrm{C}$ for $72-96$ hours (i.e. middle of typical torpor bout), and IBE when $\mathrm{T}_{\mathrm{b}}$ was $37 \pm 1^{\circ} \mathrm{C}$ for at least 3 hours. All torpid animals were sampled at 8:30am $\pm 30 \mathrm{~min}$ EST, however due to the spontaneity of IBE arousals, IBE animals were sampled between 8:30am and 10:30am EST.

Torpid animals were euthanized via cervical dislocation to prevent arousal, whereas both summer and IBE animals were euthanized by anesthetic overdose (Euthanyl: $240 \mathrm{mg} \mathrm{mL}^{-1}, 0.2 \mathrm{~mL} \cdot 100 \mathrm{~g}^{-1}$, intra-peritoneal injection). Euthanyl has no effect on mitochondrial metabolism (Takaki et al., 1997).

Immediately following euthanasia, the liver tissue was excised, weighed, and placed in ice-cold homogenization buffer (250 mM sucrose, $10 \mathrm{mM}$ HEPES, $1 \mathrm{mM}$ EGTA, $0.5 \%$ fatty acid free bovine serum albumin (BSA), $\mathrm{pH} 7.4$ at $4^{\circ} \mathrm{C}$ ) where it was cut into small pieces $\left(\sim 1 \mathrm{~mm}^{3}\right)$ using surgical scissors.

Liver tissue was then homogenized with three passes by a rotating loose fitting Teflon pestle in a $55 \mathrm{~mL}$ glass mortar at 100RPM. Next the suspension was filtered through a single piece of cheesecloth and centrifuged for $10 \mathrm{~min}$ at $1000 \mathrm{~g}$ at $4^{\circ} \mathrm{C}$ (Centrifuge 5804R, Eppendorf, Missisauga, ON). Following centrifugation, floating lipid 
was aspirated, the supernatant was poured through 4 layers of cheesecloth, and the pellet discarded. The supernatant was again centrifuged for $10 \mathrm{~min}$ at $1000 \mathrm{~g}$ at $4^{\circ} \mathrm{C}$. Once again, floating lipid was aspirated, the supernatant was poured through 4 layers of cheesecloth, and the pellet discarded. Then, the supernatant was centrifuged at $8700 \mathrm{~g}$ for $10 \mathrm{~min}$ at $4^{\circ} \mathrm{C}$. Following centrifugation, the supernatant was aspirated leaving a crude mitochondrial pellet, which was re-suspended in $1 \mathrm{~mL}$ of homogenization buffer.

Purification of the crude mitochondrial pellet was achieved using Percoll density gradient centrifugation (adapted from Petit et al., 1990). The crude mitochondrial suspension was layered on top of a Percoll gradient consisting of $10 \mathrm{~mL}$ each of $10,18,30$ and $70 \%$ layered Percoll solutions (diluted by homogenization buffer) and centrifuged at $13500 \mathrm{~g}$ for $35 \mathrm{~min}$. at $4^{\circ} \mathrm{C}$. Purified mitochondria collected at the interface between the 30 and 70\% Percoll layers, and were removed and re-suspended in homogenization buffer, and centrifuged at $8700 \mathrm{~g}$ for $10 \mathrm{~min}$ at $4^{\circ} \mathrm{C}$ to wash away residual Percoll. This process was repeated a second time on the resulting mitochondrial pellet. Finally, the resulting pellet was suspended in $1 \mathrm{~mL}$ homogenization buffer and kept on ice until assayed (maximum 7 hours). Armstrong and Staples (2010) have shown that this process of mitochondrial purification removes $85-96 \%$ of contaminating organelles (such as endoplasmic reticulum, peroxisomes, plasma membrane and lysosomes) compared with the crude pellet.

\subsubsection{Mitochondrial respiration.}

Mitochondrial respiration rates were measured using a Clark-type oxygen electrode (Oxygraph-2k, Ouroboros instruments, Innsbruck, Austria), in 2mL of MiR05 solution (0.5mM EGTA, $3 \mathrm{mM} \mathrm{MgCl}_{2} \cdot 6 \mathrm{H}_{2} \mathrm{O}, 60 \mathrm{mM} \mathrm{K}$-lactobionate, $20 \mathrm{mM}$ taurine, 
$10 \mathrm{mM} \mathrm{KH}_{2} \mathrm{PO}_{4}, 20 \mathrm{mM}$ HEPES, $110 \mathrm{mM}$ sucrose, $1 \mathrm{~g} \mathrm{~L}^{-1}$ fatty acid free BSA, $\mathrm{pH} 7.0$ at $37^{\circ} \mathrm{C}$ ) at $37^{\circ} \mathrm{C}$, while undergoing constant stirring (750 RPM). The oxygen electrodes were calibrated to oxygen-depleted MiR05 solution (using a yeast suspension to consume all present oxygen) and to air-saturated MiR05 solution, using oxygen contents previously reported (Gnaiger, 1983), and corrected for local atmospheric pressure. All substrates and inhibitors were dissolved in MiR05 solution (with the exception of rotenone and oligomycin, which were dissolved in ethanol) and added to the chamber using a Hamilton syringe. All concentrations given represent the final concentration present in the $2 \mathrm{~mL}$ Oxygraph-2k chamber during respiration rate measurements.

Both succinate-fuelled state 3 (phosphorylating) and state 4 (nonphosphorylating) respiration were measured in purified liver mitochondria (protein concentrations $2.17-8.38 \mathrm{mg} \mathrm{mL}^{-1}$ ). Initially $10 \mu$ of purified mitochondria were added to the Oxygraph-2k chamber. Rotenone $(0.5 \mu \mathrm{M})$, an ETC complex I inhibitor, was then added to the mitochondrial suspension to prevent reverse electron flow, followed by succinate $(10 \mu \mathrm{M})$ which then exclusively fuels respiration through ETC complex II (Figure 1.3). Once a steady rate of state 2 respiration was achieved ADP $(0.1 \mathrm{mM})$ was introduced into the mitochondrial suspension, stimulating state 3 respiration. Respiration rates were allowed to reach a steady state before state 3 respiration was terminated with the addition of oligomycin (an ATPase inhibitor which blocks the proton channel; $2 \mu \mathrm{g}$. $\mathrm{mL}^{-1}$ ethanol) to the mitochondrial suspension, providing an estimation of state 4 respiration rate. 


\subsubsection{Butylmalonate.}

The effect of butylmalonate (a known inhibitor of the DCT) on succinate-fuelled state 3 respiration rates was measured to determine the consequence of inhibiting the DCT. Purified mitochondria $(10 \mu \mathrm{l})$ were introduced to the Oxygraph-2k chamber, followed by the addition of butylmalonate $(200 \mu \mathrm{M}$, a concentration determined by titration to maximally inhibit respiration (Ventura et al., 2005)). Succinate-fuelled state 3 respiration rates were then established as above (section 2.5.1), and allowed to reach a steady state, after which oligomycin was introduced to the mitochondrial suspension, and state 4 respiration rates were recorded. Respiration rates from the above treatment with butylmalonate were compared with control treatments (identical to the experimental treatment, but receiving equivalent volumes of MiR05 buffer solution in place of butylmalonate), to determine the percent of inhibition attributable to butylmalonate.

\subsubsection{Palmitoyl CoA and Carnitine.}

The effect of palmitoyl CoA (both alone, and in combination with carnitine) on the respiration rates of liver mitochondria was compared among the different hibernation groups. Once steady-state state 3 respiration was established palmitoyl CoA $(2.5 \mu \mathrm{M}$, a concentration that caused maximal inhibition of state 3 respiration in preliminary experiments, see figure 3.7) was introduced to the mitochondrial suspension. Respiration rates were allowed to stabilize, and then carnitine $(5 \mathrm{mM}$, the same concentration used by Lerner et al., (1972) and Pande and Blanchaer (1971) in their work using carnitine to reverse inhibition of the adenine nucleotide transporter caused by palmitoyl CoA, preliminary experiments showed carnitine alone had no significant effects on state 3 respiration, appendix B.2) was introduced. Respiration rates were once again allowed to 
reach a steady state before state 3 respiration was terminated with the addition of oligomycin (figure 2.1). Control treatments (identical to the experimental treatment, but receiving equivalent volumes of MiR05 buffer solution in place of Palmitoyl CoA and carnitine) were performed in tandem (using an oxygraph with two chambers) with the experimental treatment to gauge any dilution or time effects.

To determine whether carnitine alone has any effect on state 3 respiration, succinate-fuelled state 3 respiration rates were established as above, and once a steady rate of state 3 respiration was measured, carnitine $(5 \mathrm{mM})$ was introduced to the mitochondrial suspension. Once again a control treatment (identical to the experimental treatment, but receiving equivalent volumes of MiR05 buffer solution in place of carnitine) was run in tandem (using an oxygraph with two chambers) with the experimental treatment. In both treatments, respiration rates were allowed to reach a steady state, and then state 4 respiration was established with the addition of oligomycin.

\subsection{Protein concentrations.}

Respiration rates were calculated relative to mitochondrial protein concentration as determined by Bradford assay (Bradford, 1976). Briefly, $10 \mu \mathrm{L}$ of each purified mitochondrial sample was diluted in $390 \mu \mathrm{L}$ of homogenization buffer and sonicated for 3 seconds, maintained on ice for 30 seconds and then sonicated again for 3 seconds (VirTis, Virsonic100; Stone Ridge, NY). Then $10 \mu \mathrm{L}$ of the sonicated mitochondrial sample was added to $200 \mu \mathrm{L}$ of Bradford assay solution (1 part Bradford protein assay solution to 3 parts homogenization buffer; Bio-Rad, Mississauga, ON) in triplicate on a 96 well plate and allowed to react for $35 \mathrm{~min}$. Protein standards containing BSA dissolved in homogenization buffer were applied (in triplicate) to the same plate, and absorbance 


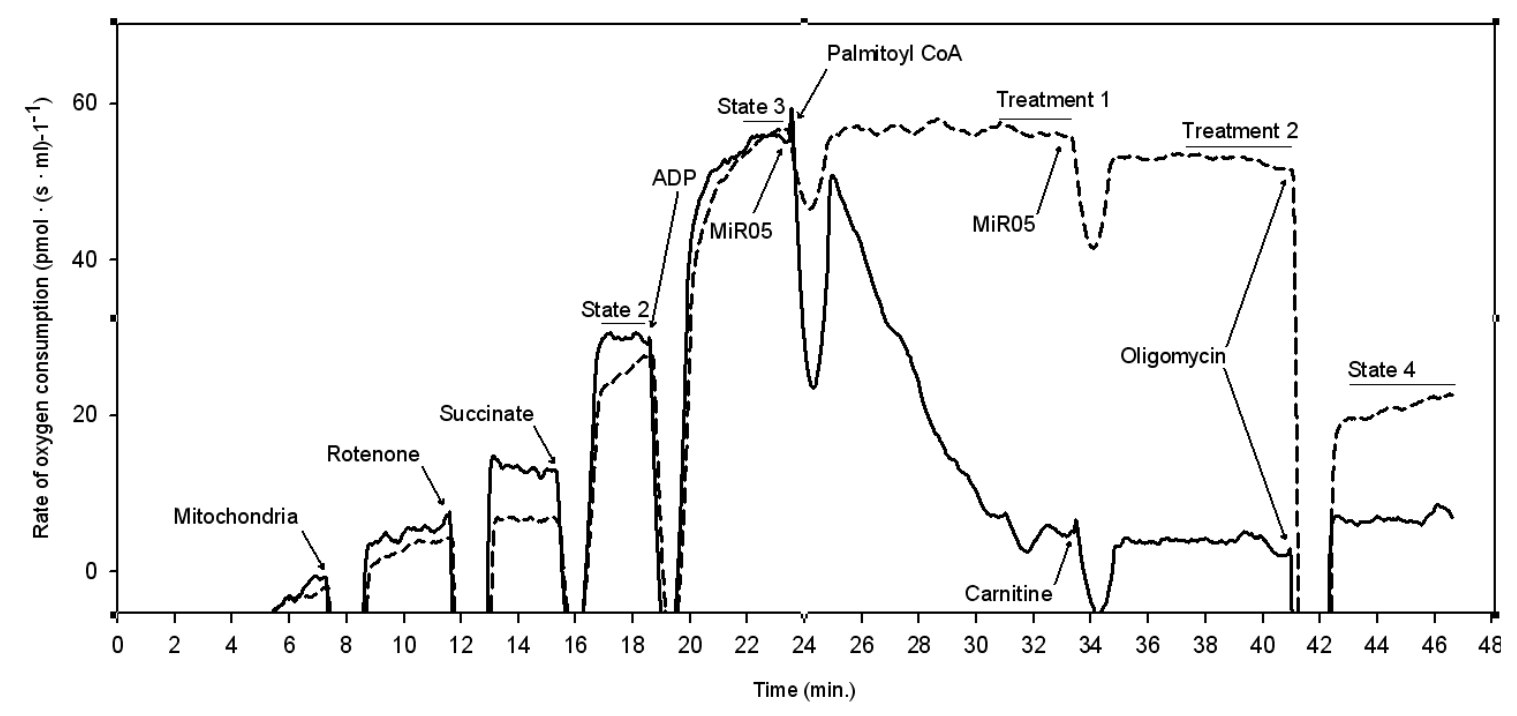

Figure 2.1 The effects of palmitoyl CoA and carnitine on succinate-fuelled mitochondrial respiration. This representative example shows the respiration rates of purified liver mitochondria ( $0.058 \mathrm{mg}$ protein) from a torpid 13 -lined ground squirrel (Ictidomys tridecemlineatus) measured by Clark-type oxygen electrode. The solid line represents treatment with palmitoyl $\mathrm{CoA}$ and carnitine, while the dashed line represents a control treatment run in tandem, using equivalent volumes of MiR05 buffer in place of the Palmitoyl CoA and carnitine additions. Arrows indicate where an addition to each chamber was made. Horizontal lines indicate where a steady rate of respiration was measured. 
(595nm) was measured using a spectrophotometer (SpectraMax 340PC, Molecular

Devices, Toronto, ON). The absorbance of a blank (containing $210 \mu \mathrm{L}$ Bradford assay solution, but no mitochondrial sample) was subtracted from each value. The mean absorbance of each standard was plotted against its concentration, and a regression line calculated. This regression was used to calculate the mean protein concentration of each sample by its absorbance.

\subsection{Carnitine-palmitoyl transferase activity assay.}

To determine the activity of carnitine-palmitoyl transferase from frozen mitochondrial samples, $100 \mu \mathrm{L}$ of purified mitochondrial suspension was combined with $900 \mu \mathrm{L}$ glycerol buffer $\left(20 \mathrm{mM} \mathrm{Na}_{2} \mathrm{HPO}_{4}, 0.5 \mathrm{mM}\right.$ EDTA, 0.2\% BSA (W/V), 50\% glycerol (V/V), $0.1 \%$ triton $\mathrm{X}-100(\mathrm{~V} / \mathrm{V}), \mathrm{pH} 7.4$ at $\left.18^{\circ} \mathrm{C}\right)$, and sonicated 3 times for 10 seconds, with 30 seconds rest on ice between sonication bursts. For each sample $725 \mu \mathrm{L}$ of TRIS buffer (19.47 mM TRIS HCl, $30.54 \mathrm{mM}$ TRIS Base, $\mathrm{pH} 8.00$ at $37^{\circ} \mathrm{C}$ ), $50 \mu \mathrm{L}$ $0.15 \mathrm{mM}$ DTNB solution, $100 \mu \mathrm{L} 0.035 \mathrm{mM}$ palmitoyl CoA solution, $100 \mu \mathrm{L}$ of $5 \mathrm{mM}$ carnitine solution and $25 \mu \mathrm{L}$ mitochondrial homogenate (ranging from 0.054-0.209 mg protein) were combined in a cuvette. The cuvette was covered by parafilm, gently inverted to mix, and absorbance at $412 \mathrm{~nm}$ was measured using a spectrophotometer for a period of $120 \mathrm{~s}$ at $37^{\circ} \mathrm{C}$. A blank control determination was also made in the absence of carnitine.

Enzyme activity was calculated using the following formula:

$$
\mathrm{U} / \mathrm{mg} \text { protein }=\frac{\left(\text { slope }_{\text {Carnitine }}-\text { slope }_{\text {Control }}\right) * 1000 \mu \mathrm{L} * \mathrm{D}}{\varepsilon * 25 \mu \mathrm{L} * \mathrm{P}}
$$


Where $\mathrm{U}$ is units of enzyme activity in $\mu \mathrm{mol}$ of substrate converted to

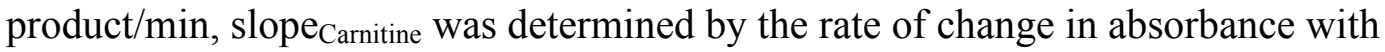
carnitine, and slope Control $_{\text {was }}$ the rate of change in absorbance without carnitine, $1000 \mu \mathrm{L}$ is the volume of the cuvette, D is the dilution factor of the mitochondrial suspension (10), $\varepsilon$ is the extinction coefficient of DTNB (13.6), $25 \mu \mathrm{L}$ is the volume of the sample, and P is the mitochondrial protein concentration of the original suspension.

\subsection{Protein Quantification by Western blotting.}

To further characterize any changes in mitochondrial respiration among hibernation states, I quantified subunits of ETC complexes using Western blotting. To prepare samples, protein concentrations of all mitochondrial suspensions were determined by Pierce bicinchroninic acid (BCA) protein assay kit (Thermo Scientific), and $33 \mu \mathrm{g}$ of purified mitochondrial protein was suspended in $60 \mu \mathrm{L} \mathrm{ddH}_{2} \mathrm{O}, 25 \mu \mathrm{L}$ NuPAGE LDS sample buffer (Invitrogen) and $15 \mu \mathrm{L}$ NuPAGE Sample Reducing Agent (Invitrogen) to a total volume of $100 \mu \mathrm{L}$. Samples were then heated in a water bath for 5 minutes at $\sim 37^{\circ} \mathrm{C}$, after which $8 \mu \mathrm{L}$ of sample $(2.64 \mu \mathrm{g}$ protein, a quantity determined by a preliminary experiment which used $5.2 \mu \mathrm{g}$ of protein, and caused over-saturation of antibody signal, thus the quantity was reduced by half to increase resolution) was loaded onto NuPAGE Novex 4-12\% Bis-Tris Gels (1.5-mm thick, 15-well, Invitrogen). Samples were separated in MES-SDS running buffer (50mM 2-(N-morpholino) ethanesulfonic Acid, 50mM TRIS (hydroxymethyl) aminomethane, $0.1 \%$ sodium dodecyl sulphate, $1 \mathrm{mM}$ EDTA) combined with $500 \mu \mathrm{L}$ of $2 \mathrm{M} \mathrm{Na}_{2} \mathrm{~S}$, at $160 \mathrm{~V}$ for 1 hour. Proteins were then transferred to nitrocellulose membranes at $100 \mathrm{~V}$ for 2hours. After which membranes 
were blocked overnight at $4^{\circ} \mathrm{C}$ in $5 \%$ BSA Tris Buffered Saline with the detergent Tween-20 (TBST; 150mM NaCl 25mM Tris, 4mM KCl, 0.1\% Tween-20).

Following blocking with BSA (5\% BSA W/V in TBST), membranes were treated with the MitoProfile Total OXPHOS Rodent Antibody Cocktail (Complex I subunit NDUFB8 (NADH dehydrogenase 1 beta subcomplex 8); Complex II subunit 30kDa; complex III Core protein 2; Complex IV subunit I; ATP synthase alpha subunit; ab110413, host: mouse; Mitosciences) at a 1:1000 dilution in 5\% BSA TBST for 2hours at room temperature. While this antibody cocktail is used routinely in laboratory rodents such as mice (Hoy et al., 2011) and rats (Rector et al., 2011), there are unfortunately no antibodies specific to 13-lined ground squirrels.

I used voltage dependent anion channel protein 1 (VDAC1) as a loading control, as it does not differ among metabolic states (Chung, 2013), and was used previously as a loading control in 13-lined ground squirrels in the work of Xu et al. (2013) and Chung et al. (2013). Membranes were prepared as above, and were then treated with AntiVDAC/Porin primary antibody (ab14734, host: mouse; Mitosciences) at 1:1000 dilution in $5 \%$ BSA TBST for 2 hours at room temperature.

Membranes were then washed 4 times with TBST for 10 minutes, and then treated with a donkey anti-mouse horseradish peroxidase secondary antibody at a 1:5000 dilution in 5\% BSA TBST for 1 hour at room temperature. Membranes were then washed 4 times with TBST for 10 minutes.

Membranes were treated with enhanced chemiluminescence reagent (Luminata Forte Western HRP Substrate, Millipore). Immunoreactive bands were imaged with a 
VersaDoc Imaging System (Bio-Rad, Richmond, CA). Densitometry was performed with Image Lab Software 3.0 (Bio-Rad).

\subsection{Cytochrome C oxidase activity assay.}

The activity of cytochrome $\mathrm{C}$ oxidase from frozen mitochondrial samples was determined to further characterize mitochondrial respiration. This activity was determined using $10 \mu \mathrm{L}$ of purified mitochondrial suspension combined with $200 \mu \mathrm{L}$ double distilled water and sonicated 3 times for 10 seconds, with 30 seconds rest on ice in-between. Each sample was prepared in triplicate on a 96 -well plate with $272 \mu \mathrm{L}$ phosphate buffer $\left(10 \mathrm{mM} \mathrm{KH}_{2} \mathrm{PO}_{4} \mathrm{pH} 7.4\right.$ at $\left.37^{\circ} \mathrm{C}\right), 25 \mu \mathrm{L}$ of Cytochrome C solution $\left(0.40 \mathrm{mM}\right.$ cytochrome $\mathrm{C}$, acidified with $10 \mu \mathrm{L} 1 \mathrm{M} \mathrm{HCl}$ and reduced by crystals of $\left.\mathrm{NaBH}_{4}\right)$ and $12 \mu \mathrm{L}$ mitochondrial suspension. Absorbance at $550 \mathrm{~nm}$ was measured at $37^{\circ} \mathrm{C}$, for 2 minutes at 10 second intervals, using a spectrophotometer.

Enzyme activity was calculated using the following formula:

$$
\mathrm{U} / \mathrm{mg} \text { protein }=\frac{(\Delta \mathrm{A} / \mathrm{min}) * \mathrm{D} * 309 \mu \mathrm{L}}{\varepsilon * 12 \mu \mathrm{L} * \mathrm{P}}
$$

Where $\mathrm{U}$ is the $\mu \mathrm{mol}$ of substrate converted to product $/ \mathrm{min}, \Delta \mathrm{A} / \mathrm{min}$ is the change in absorbance at 550nm per minute, $\mathrm{D}$ is the dilution factor of the mitochondrial suspension (20), $390 \mu \mathrm{L}$ is the total reaction volume in the well, $\varepsilon$ is the extinction coefficient of cytochrome $\mathrm{C}\left(27.7 \mathrm{mM}^{-1} \mathrm{~cm}^{-1}\right), 12 \mu \mathrm{L}$ is the volume of the sample, and $\mathrm{P}$ is the mitochondrial protein concentration of the original suspension (mg protein/mL, as determined by Bradford assay). 


\subsection{Data Analysis.}

All data presented are mean \pm SEM. In all cases $\mathrm{P}<0.05$ was considered statistically significant. Differences in succinate-fuelled state 3 and 4 respiration rates among different metabolic states were analyzed using a one-way ANOVA and post hoc Tukey's test. Differences in succinate-fuelled state 3 and 4 respiration rates among metabolic states and within treatment groups (palmitoyl CoA, butylmalonate, carnitine, palmitoyl-carnitine) were analyzed using a two-way ANOVA and post hoc LSD test (percent values were arcsine transformed prior to statistical testing). Differences in enzyme activity among metabolic states were analysed by one-way ANOVA with post hoc Tukey's test. Differences in protein abundance as determined by Western blotting were analyzed by one-way ANOVA and post-hoc Tukey's test. All statistical analyses were completed using SPSS 20.0 (IBM, Armonk NY). 


\section{CHAPTER 3}

\section{RESULTS}

\subsection{Whole animal characteristics.}

Whole animal body weight was measured weekly during cage changes during the summer (May-October). Once hibernation began, animals were left undisturbed, however animals sampled throughout the hibernation season (January-March) were weighed prior to sampling. A pattern of weight gain during summer months followed by weight loss during the hibernation season was observed (figure 3.1).

The $\mathrm{T}_{\mathrm{b}}$ of all animals was monitored using radio telemetry implants. As animals entered the hibernation season, periods of torpor $\left(\mathrm{T}_{b}=\sim 5^{\circ} \mathrm{C}\right)$ were observed, lasting from 5-14 days, interrupted by short periods ( $\sim 8$ hours $)$ of $\operatorname{IBE}\left(\mathrm{T}_{\mathrm{b}}=\sim 37^{\circ} \mathrm{C}\right)$, which alternated cyclically throughout the hibernation season (figure 3.2).

\subsection{Mitochondrial respiration.}

State 3 succinate-fuelled liver mitochondrial respiration was not significantly different between Summer and IBE groups $(\mathrm{P}=0.974)$. There was significant suppression of State 3 respiration in torpid animals when compared with both IBE and SA (48 and 44\% respectively; $\mathrm{P}<0.05$, figure 3.3 ). The ANOVA reported a significant difference between state 4 succinate-fuelled respiration $(\mathrm{P}<0.05$, figure 3.3$)$, however a post hoc Tukey's test did not find any significant pair-wise differences among groups, although torpor was nearly significantly different from summer $(\mathrm{P}=0.055)$.

It has been assumed that any apparent differences among metabolic states are the result of changes in the activity of oxidative phosphorylation enzymes. However, an alternative explanation is that the concentration of mitochondrial proteins that do not 


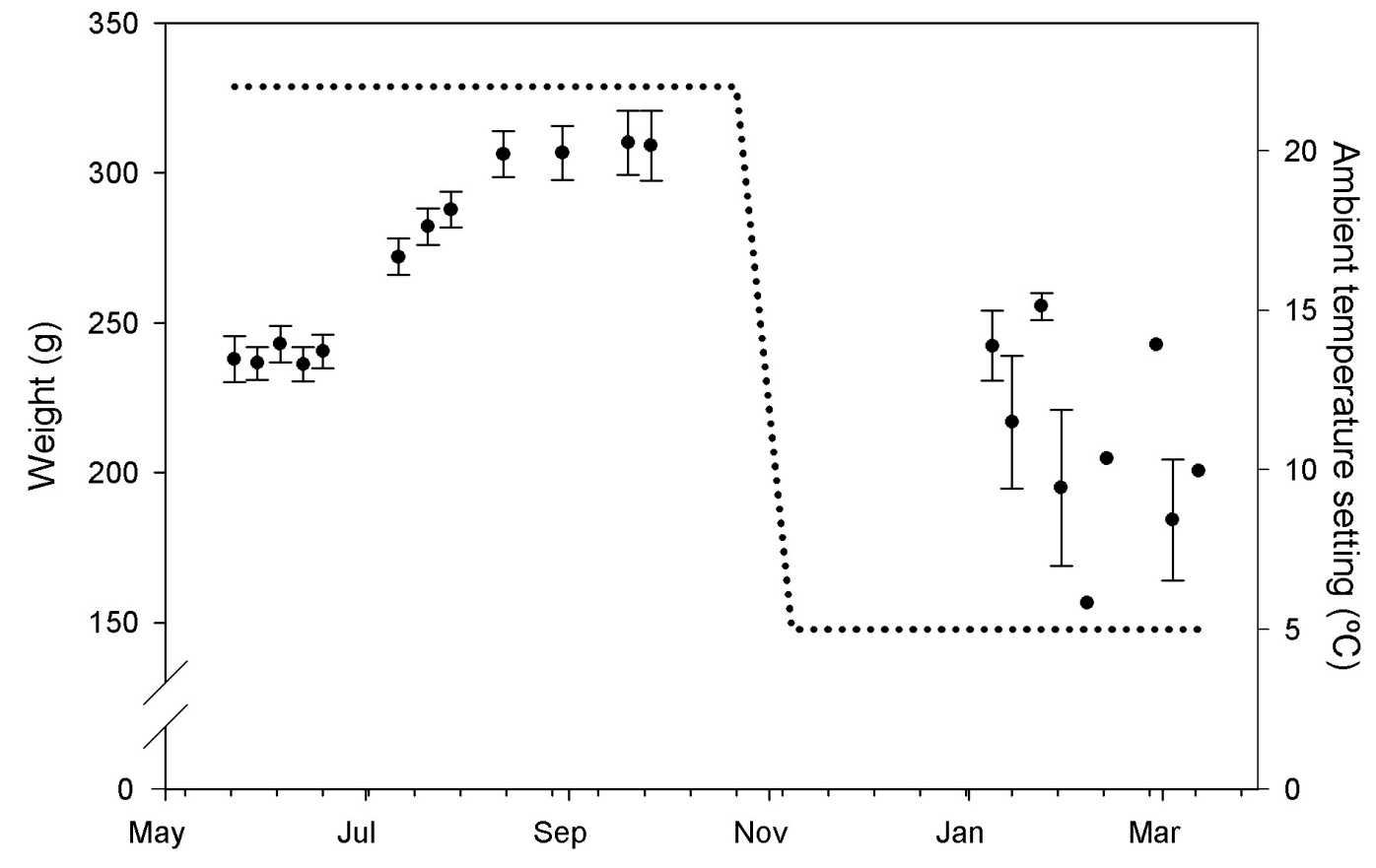

Figure 3.1 Mass of 13-lined ground squirrels (Ictidomys tridecemlineatus) from May 2012-March 2013. Data are means \pm SEM, (except in cases of $n=1$ ). Weights of summeractive squirrels were measured weekly during cage changes from May 2012-October $2012(\mathrm{n}=9-24)$. Weights of winter squirrels (torpid and IBE) were recorded following euthanasia throughout January 2013-February $2013(n=1-4$, squirrels are pooled by the week in which they were sampled). Dashed line indicates ambient temperature of the chamber the animals were housed in. 


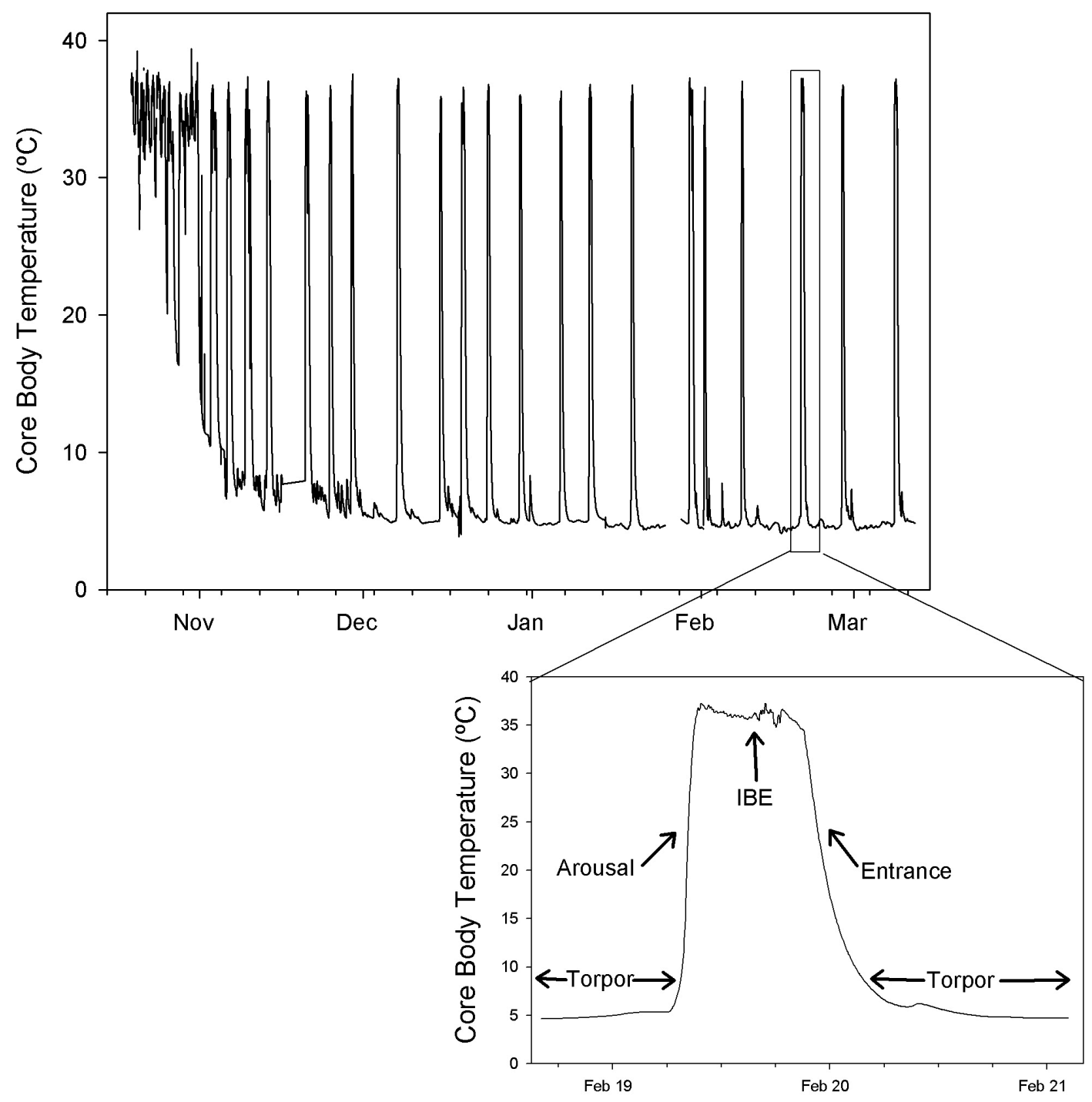

Figure 3.2 Core body temperature of a hibernating 13-lined ground squirrel (Ictidomys tridecemlineatus) measured by a temperature sensing radiotelemetry implant in the present study. Top panel shows the core body temperature of an individual animal over the course of the hibernation season, showing repetitive cycling between torpor and inter-bout euthermia (IBE). Cutaway shows the core body temperature of the same individual undergoing a single interbout arousal, beginning in torpor, followed by arousal, IBE and concluding with entrance back into torpor. 


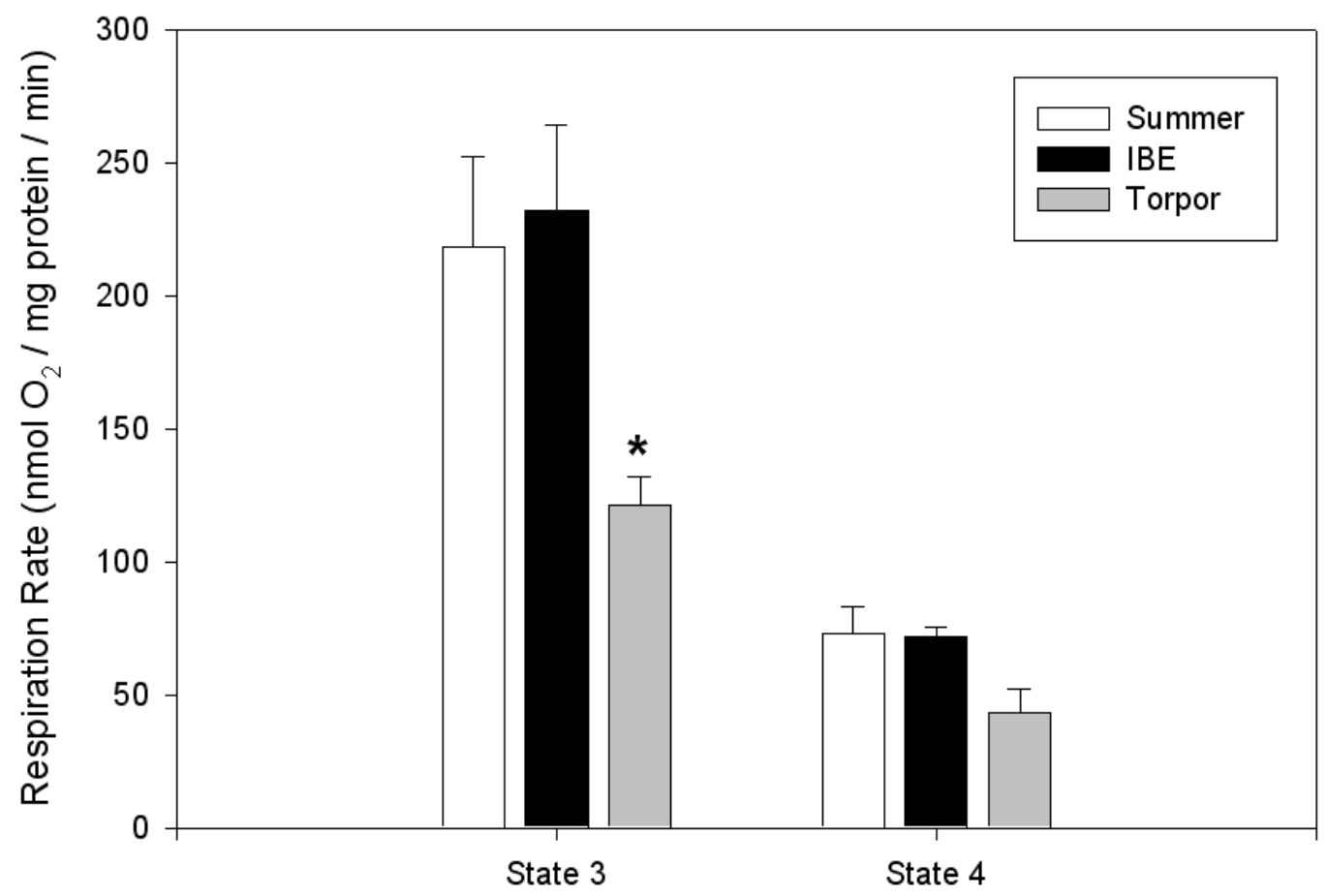

Figure 3.3 Succinate-fuelled State 3 and State 4 respiration rates of liver mitochondria of summer, interbout euthermic (IBE) and torpid 13-lined ground squirrels measured in vitro at $37^{\circ} \mathrm{C}$. Data are means \pm SEM. Asterisk indicates significant difference among metabolic states (IBE $n=5$, summer $n=6$, torpid $n=6, P<$ $0.05)$, as determined by one-way ANOVA. 
contribute to oxidative phosphorylation (eg. urea cycle enzymes) change among metabolic states, causing respiration, expressed relative to total mitochondrial protein, to change as well. To differentiate between these possibilities we standardized respiration rate to the activity of cytochrome C oxidase, an ETC enzyme that does not display differential regulation by phosphorylation among metabolic states (Chung et al., 2013). The activity of cytochrome $\mathrm{C}$ oxidase was measured in liver mitochondria purified from summer, IBE and torpid animals. If the decline in respiration rate of torpid mitochondria was due to changes in mitochondrial proteins, I would predict a similar pattern in cytochrome $\mathrm{C}$ oxidase activity (expressed per mg mitochondrial protein). However, cytochrome $\mathrm{C}$ oxidase activity did not decline in torpor, but in fact was significantly higher than either summer or IBE animals $(65 \%$ and $66 \%$ respectively, $\mathrm{P}<0.001$, figure 3.4). I then divided respiration rates (expressed relative to mitochondrial protein) by cytochrome $\mathrm{C}$ oxidase activity (also expressed relative to mitochondrial protein), yielding respiration rates relative to cytochrome $\mathrm{C}$ oxidase activity, thereby eliminating any effect of mitochondrial protein content. When respiration was expressed this way, state 3 respiration was not significantly different between Summer and IBE $(P=0.351$; figure 3.5), but there was significant suppression in torpid animals when compared with both IBE and SA (67 and $60 \%$ respectively; $\mathrm{P}<0.05$; figure 3.5). State 4 succinate-fuelled liver mitochondrial respiration was also significantly lower in torpor than in summer or IBE animals $(\mathrm{P}<0.05$, figure 3.5$)$. 


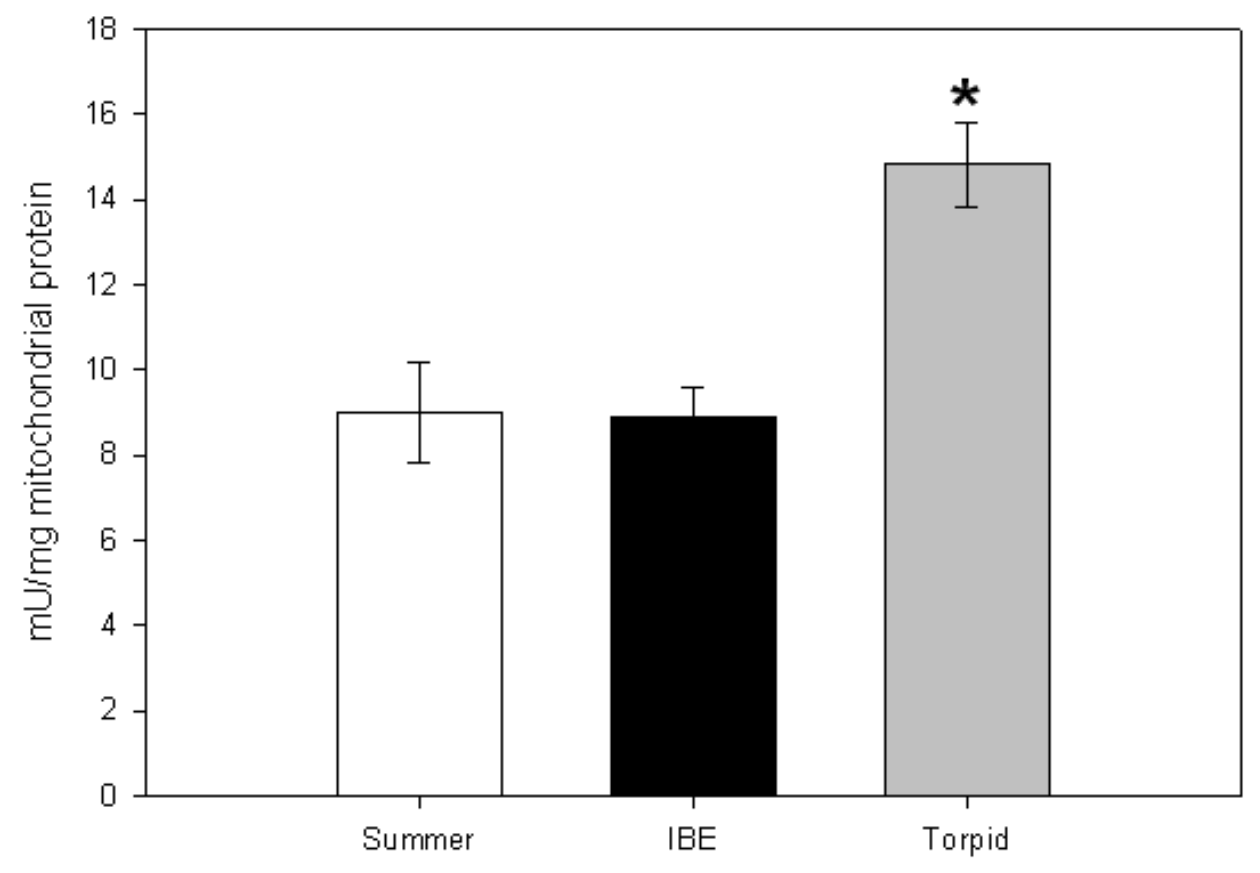

Figure 3.4 Cytochrome $\mathrm{C}$ oxidase activity is significantly higher in torpid mitochondria than mitochondria from summer and IBE animals. The activity of cytochrome $\mathrm{C}$ oxidase $\mathrm{mU}\left(10^{-3} \mu \mathrm{mol}\right.$ of substrate converted to product $\left./ \mathrm{min}\right)$ was assessed in liver mitochondria isolated from summer, interbout euthermic (IBE) and torpid animals. Data are means \pm SEM. The asterisk indicates activity of cytochrome $\mathrm{c}$ oxidase was found to be significantly higher in torpor than summer and IBE $(\mathrm{P}<0.05)$, as assessed by one-way ANOVA and post hoc Tukey test (Summer $n=6, \operatorname{IBE} n=4$, torpid $n=6$ ). 


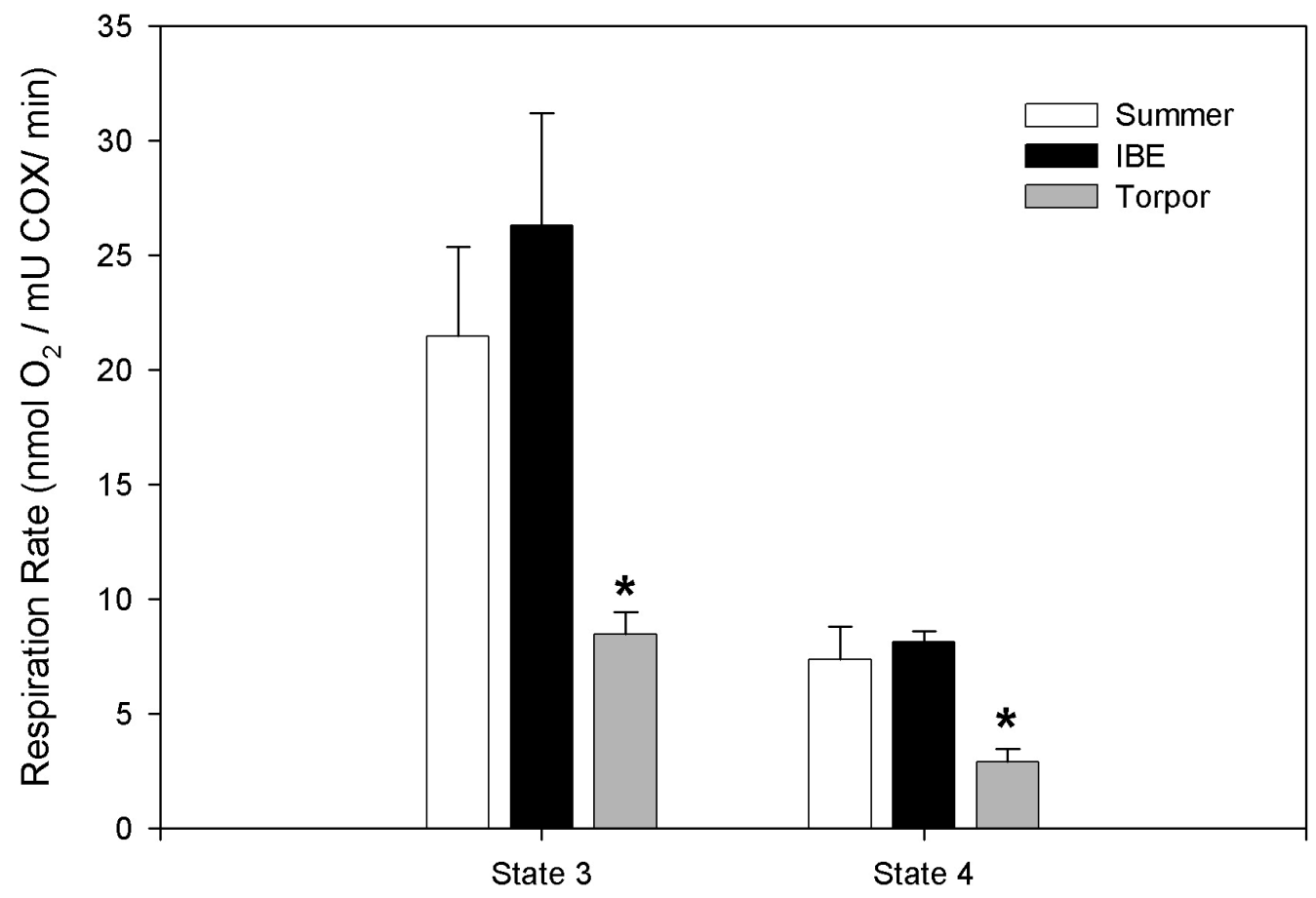

Figure 3.5 Succinate-fuelled State 3 and State 4 respiration rates relative to cytochrome c oxidase activity of liver mitochondria among metabolic states. Respiration relative to cytochrome $\mathrm{c}$ oxidase activity was assessed in liver mitochondria isolated from summer, interbout euthermic (IBE) and torpid 13-lined ground squirrels measured in vitro at $37^{\circ} \mathrm{C}$. Data are means \pm SEM. Asterisk indicates significant difference among metabolic states (IBE $n=4$, summer $n=6$, torpid $n=6, P<0.05$ ), as determined by one-way ANOVA and post hoc Tukey test. 


\subsection{No difference in ETC complexes II-V subunit abundance among metabolic states.}

To further confirm that changes in respiration seen in torpid mitochondria are due to regulatory differences among states, rather than differences in abundance in ETC proteins, the abundance of ETC complexes I through V was determined by Western blot analysis. Each ETC complex was identified with an antibody specific to one of its subunits (complex I: subunit NDUFB8, complex II: subunit 30kDa, complex III: Core protein 2, complex IV: subunit I, ATP synthase alpha subunit). Protein levels were standardized to VDAC1, an outer mitochondrial membrane housekeeping protein that did not to differ significantly among metabolic states ( $\mathrm{P}=0.376$, one-way ANOVA), confirming VDAC1 was an appropriate loading control. Analysis with a one-way ANOVA and post-hoc Tukey's test revealed that protein abundance of ETC complex I was significantly higher in IBE than in summer or torpid animals $(n=4$ for each metabolic state, $\mathrm{P}<0.05$, figure 3.6). While protein abundance of ETC complexes II, III, IV and $\mathrm{V}$ showed no significant differences in protein abundance among metabolic states (figure 3.6). As work in this thesis is conducted in the presence of the ETC complex I inhibitor rotenone, these results imply that the metabolic suppression observed in torpor is not attributable to a decrease in the abundance of this ETC protein or any ETC complex downstream of complex II. 

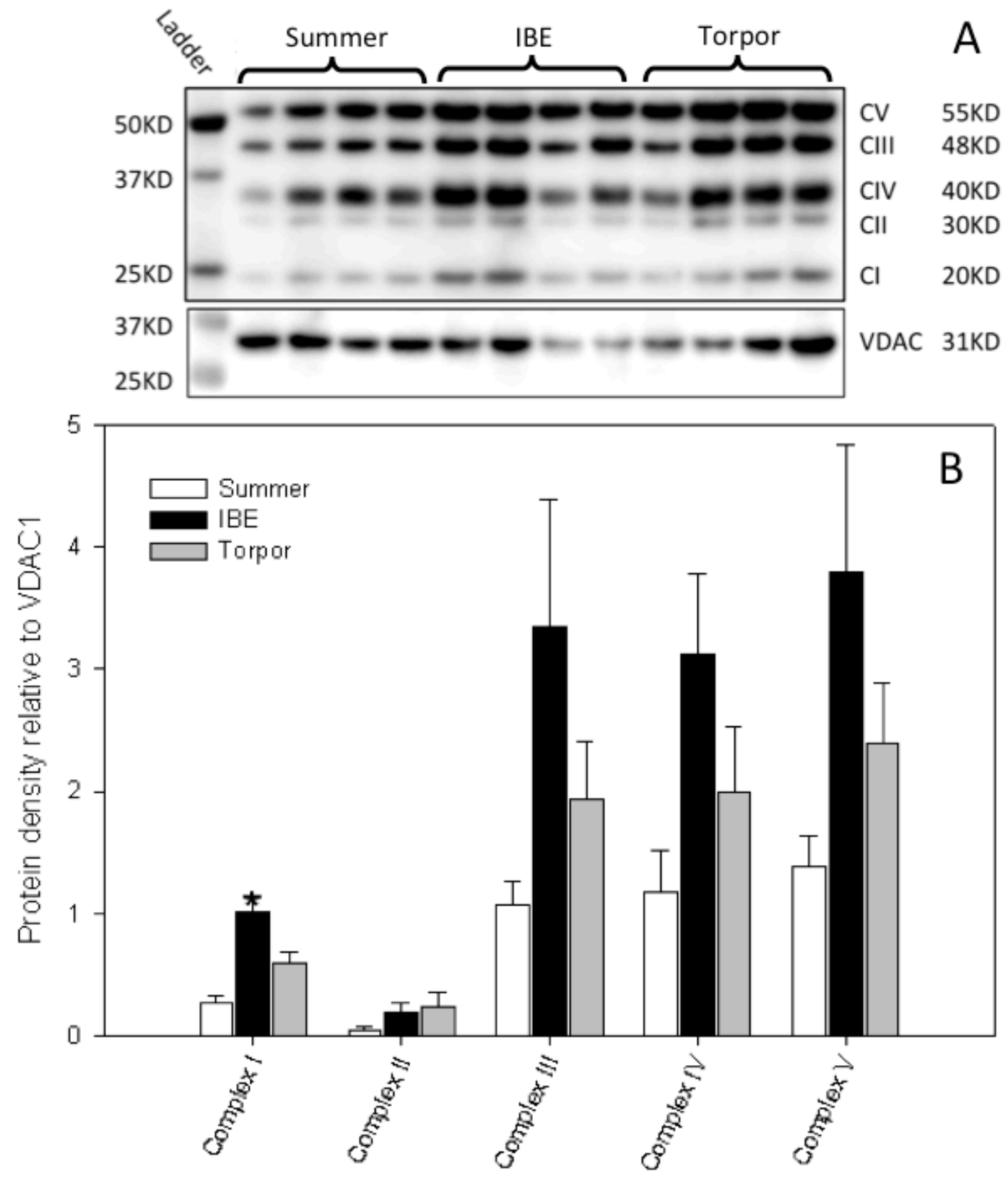

Figure 3.6 Protein abundance of electron transport chain (ETC) complexes I-V among metabolic states, analyzed by Western blot analysis. Each ETC complex was identified by an antibody specific to an associated subunit; complex I subunit NDUFB8; complex II subunit 30kDa; complex III Core protein 2; complex IV subunit I; complex V alpha subunit. Protein levels were determined by densitometry, standardized to VDAC1. Panel A shows a representative blot for ETC complexes I-V and VDAC1. Panel B shows protein abundance amongst summer, interbout euthermic (IBE) and torpid animals.

Asterisk indicates a significant difference in protein abundance among metabolic states (n $=4$ for each metabolic state, $\mathrm{P}<0.05$ ), as determined by one-way ANOVA and post hoc Tukey's test. All data presented as mean \pm SEM. 


\subsection{Suppression of mitochondrial respiration by butylmalonate, palmitoyl CoA and the effect of carnitine.}

In order to choose the concentration of palmitoyl CoA that stimulated maximal inhibition of succinate fuelled state 3 respiration, a titration of increasing concentrations of palmitoyl CoA was performed as a preliminary experiment, identifying $2.5 \mu \mathrm{M}$ as the ideal concentration (figure 3.7). At this concentration, Palmitoyl CoA caused significant suppression of succinate fuelled state 3 respiration rates in both IBE and torpid mitochondria (Figure 3.8).

State 3 respiration rates were analyzed by determining the percent of suppression relative to a paired control for each treatment. In order to normally distribute the data set, percent values were arcsine transformed prior to statistical tests. Butylmalonate (a known inhibitor of the DCT) induced significant suppression (43\% in torpor and $27 \%$ in IBE, P $<0.05$, figure 3.9). However, palmitoyl CoA induced even greater suppression from control ( $72 \%$ in torpor and $87 \%$ in IBE, $\mathrm{P}<0.05$, figure 3.9$)$ than butylmalonate. Treatment with palmitoyl CoA followed by carnitine did not elicit the predicted rescue of respiration, and respiration rate remained statistically indistinguishable from the Palmitoyl CoA treatment ( $89 \%$ in torpor and $69 \%$ in IBE, $\mathrm{P}=0.717$, figure 3.9). No significant differences were seen between metabolic states, and post hoc LSD test revealed no significant interactions.

\subsection{Effect of carnitine on mitochondrial respiration.}

Carnitine had no significant effect $(\mathrm{P}=0.269)$ on succinate fuelled mitochondrial respiration rate (figure 3.10), indicating that carnitine is unable to reverse the suppression 


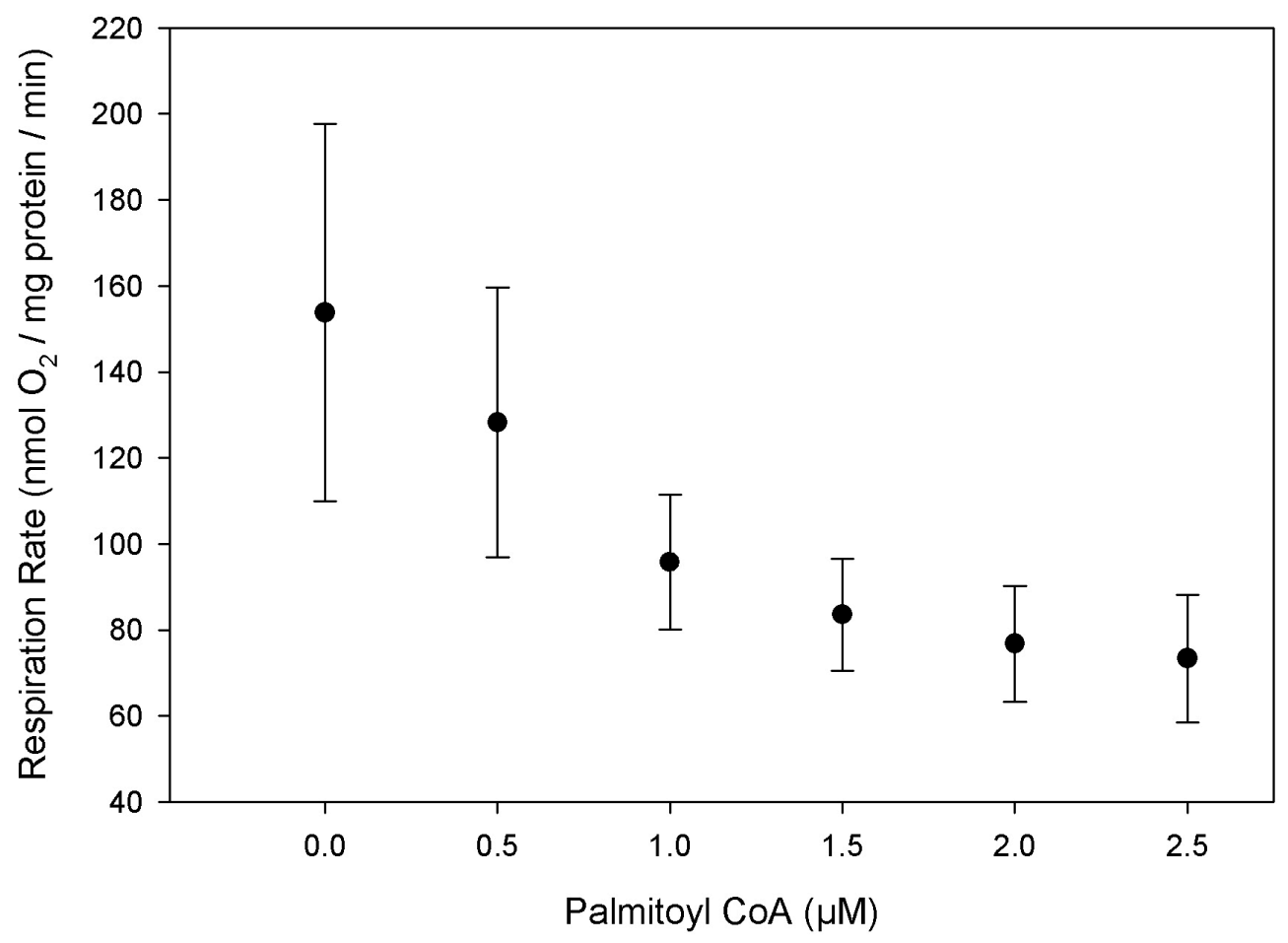

Figure 3.7 Titration of inhibitory effect of palmitoyl CoA on state 3 succinate-fuelled respiration in 13-lined ground squirrel mitochondria. Data are means \pm SEM. State 3 respiration was established in purified mitochondria from summer animals $(n=3)$, and $0.5 \mu \mathrm{M}$ additions of palmitoyl CoA were sequentially added to a final concentration of $2.5 \mu \mathrm{M}$. Respiration rates were measured when they reached a steady state following each addition. 


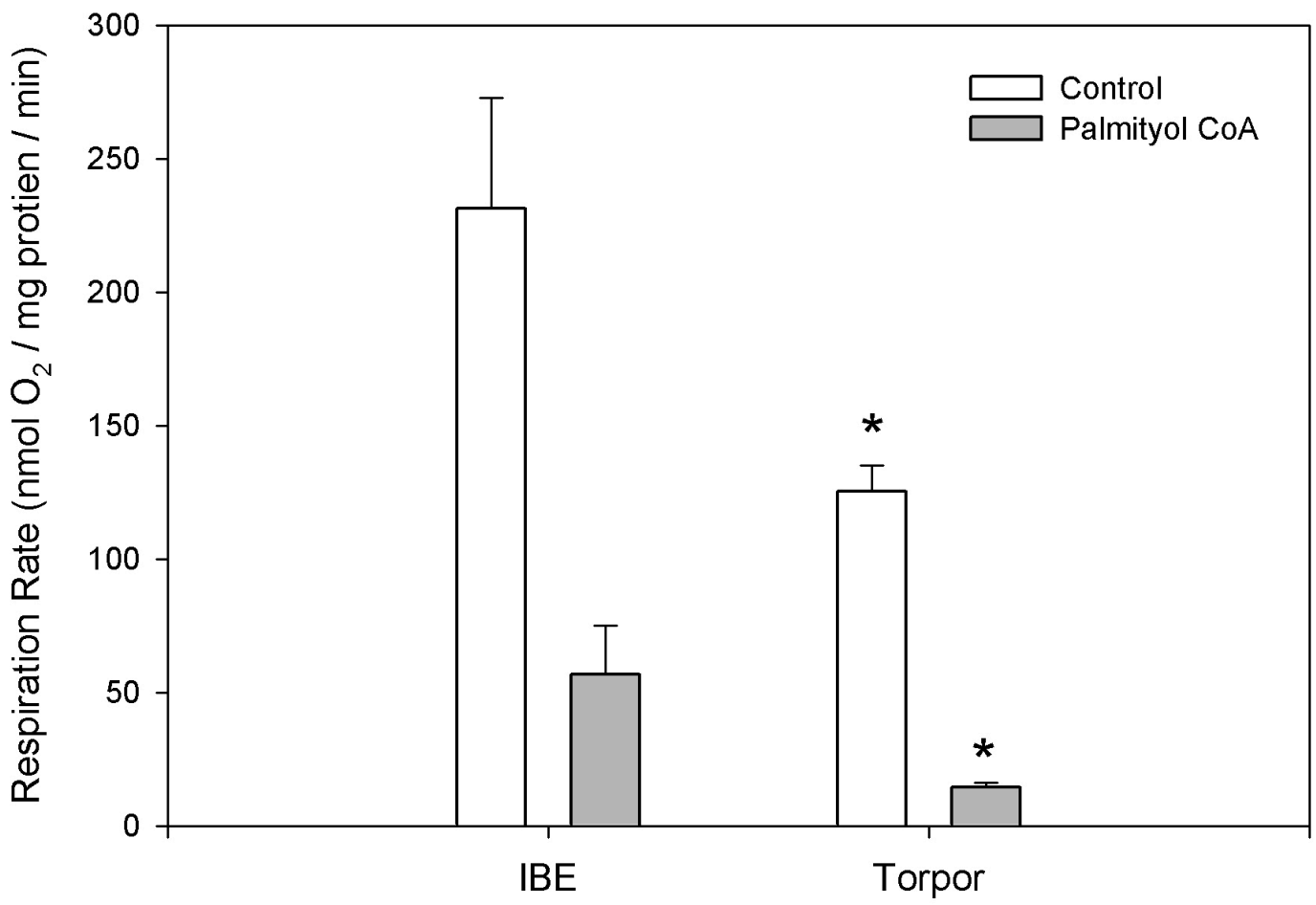

Figure 3.8 Palmitoyl CoA suppresses succinate-fuelled state 3 respiration rates of liver mitochondria of torpid and interbout euthermic (IBE) 13-lined ground

squirrels. Open bars denote the control treatment, while shaded bars display treatment with palmitoyl CoA $(2.5 \mu \mathrm{M})$. Data are means \pm SEM. Asterisks indicate a significant difference between metabolic state, in both states palmitoyl CoA significantly suppresses respiration (IBE $n=5$, torpid $n=6)$, as assessed by two-way ANOVA $(P<0.05)$. 


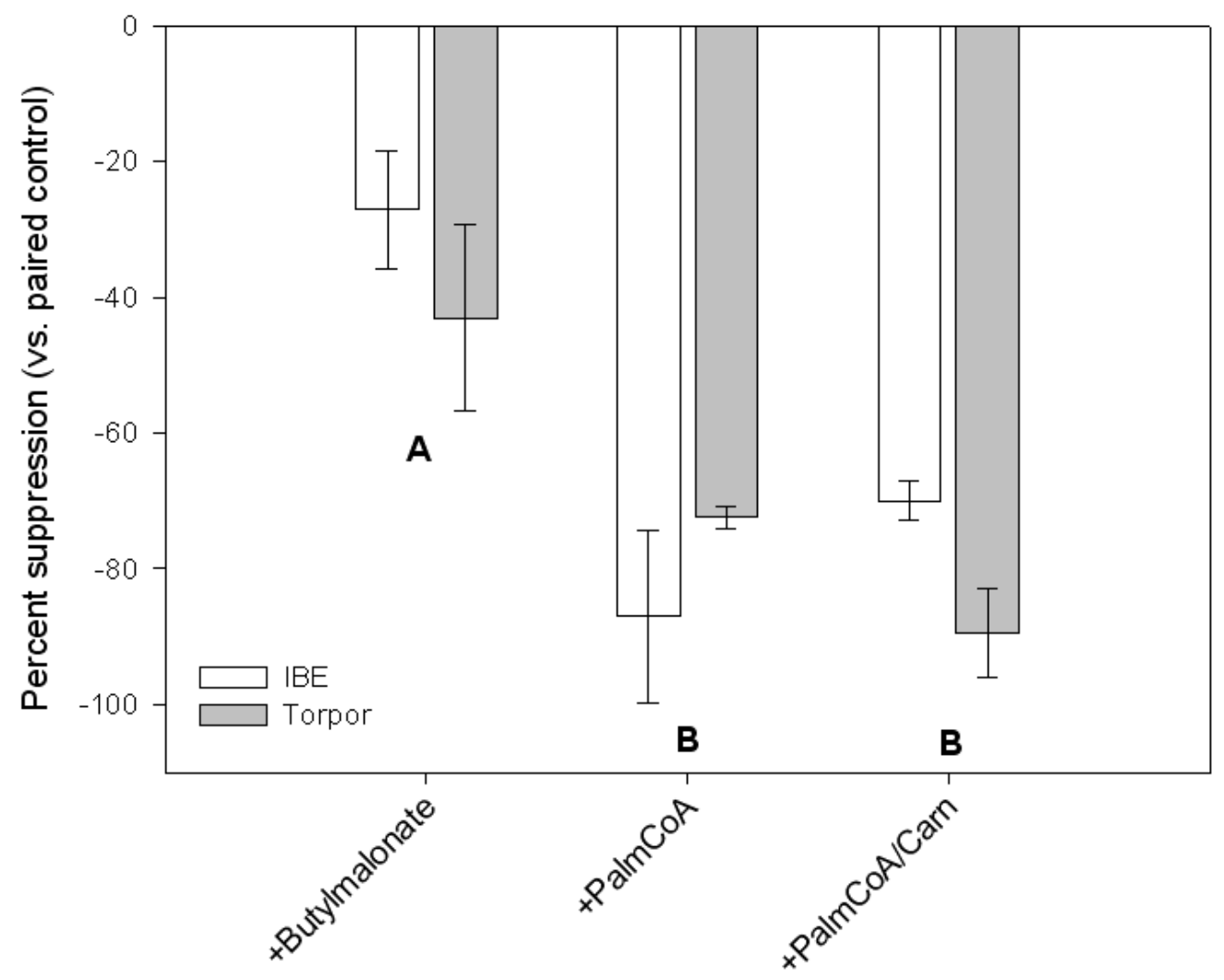

Figure 3.9 Percent suppression of respiration rate in interbout euthermic (IBE) and torpid liver mitochondria following treatment with butylmalonate, Palmitoyl CoA and Carnitine compared to paired control. Data are the mean percent suppression of the paired control \pm SEM, following additions of butylmalonate $(200 \mu \mathrm{M})$, palmitoyl CoA $(2.5 \mu \mathrm{M})$ and carnitine $(5 \mathrm{mM})$. Different letters indicate a significant difference between treatments $(\mathrm{P}<0.05)$. No significant difference was seen between metabolic state (IBE $\mathrm{n}$ $=5$, torpid $\mathrm{n}=6$ ), and post hoc least squares difference test revealed no significant interactions. 


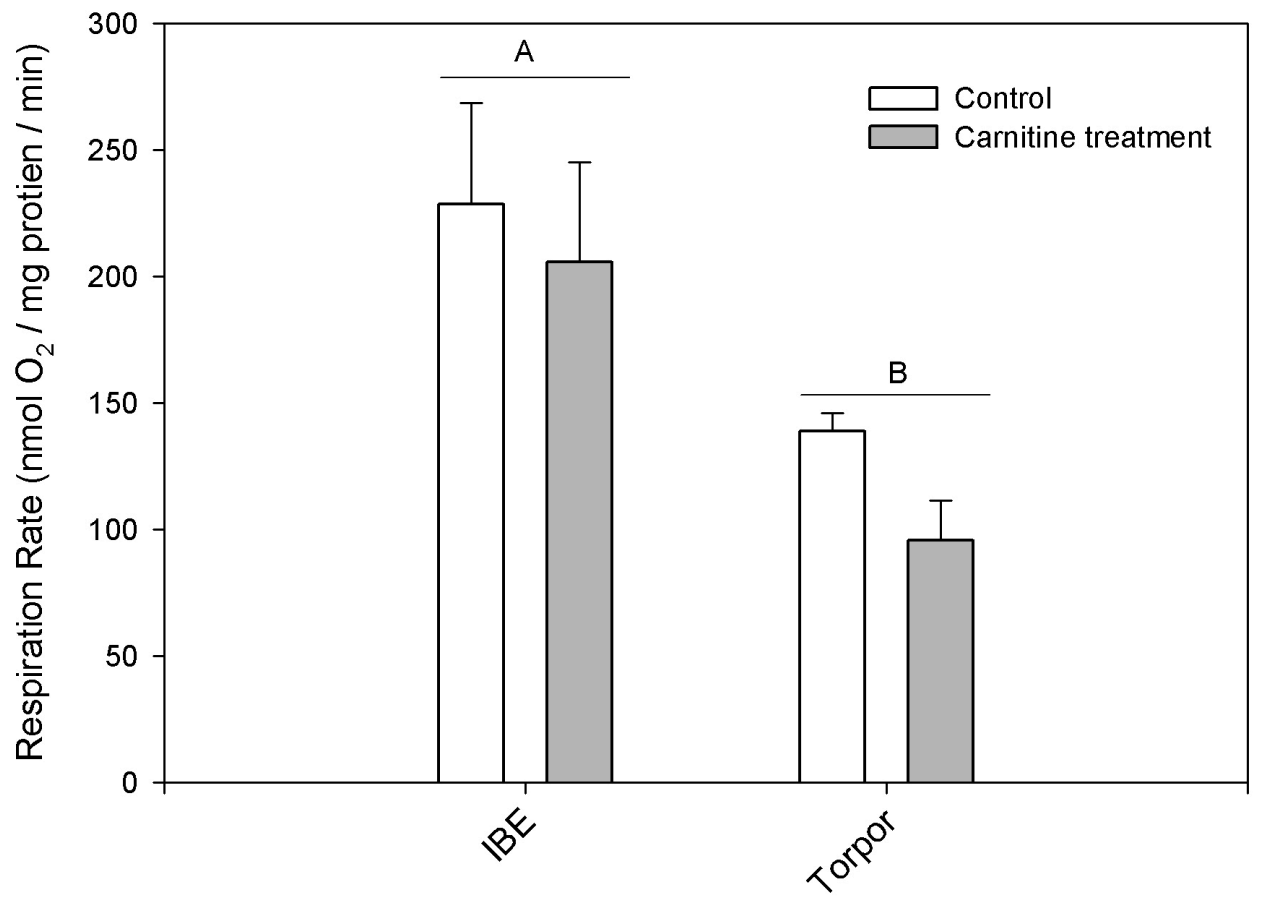

Figure 3.10 Carnitine has no rescuing effect on respiration rates of the liver mitochondria of torpid 13-lined ground squirrels. The succinate-fuelled State 3 respiration rates of liver mitochondria of interbout euthermic (IBE) and torpid animals. Open bars denote the control treatment, while shaded bars display treatment with carnitine $(5 \mathrm{mM})$. Data are means \pm SEM. Different letters depict a significant difference of respiration rate between metabolic state (IBE $n=5$, torpor $n=6$ ) No significant difference was found between treatments, indicating carnitine has no effect on mitochondrial respiration, and does not reverse the suppression observed in the liver mitochondria of torpid 13 lined ground squirrels. 
observed in the liver mitochondria of torpid 13 lined ground squirrels. Additionally, palmitoyl-carnitine (the combined product palmitoyl CoA and carnitine by CPT) had no significant effect on respiration (appendix B).

\subsection{Carnitine palmitoyl transferase activity among metabolic states.}

Carnitine was expected to increase respiration rates of torpid mitochondria or restore respiration rates of mitochondria suppressed by the addition of palmitoyl CoA, however it had no such "rescuing" effect in either case (figure 3.10 and 3.9 respectively). So in order to further explore whether CPT (the enzyme responsible for binding carnitine to palmitoyl CoA, thereby facilitating transport into the matrix by CAT) might differ among metabolic states, I assayed total CPT activity in liver mitochondria. The activity of CPT did not differ significantly among metabolic states $(\mathrm{P}=0.889$, figure 3.11$)$, suggesting that the capacity to form palmitoyl-carnitine operates consistently among metabolic states. 


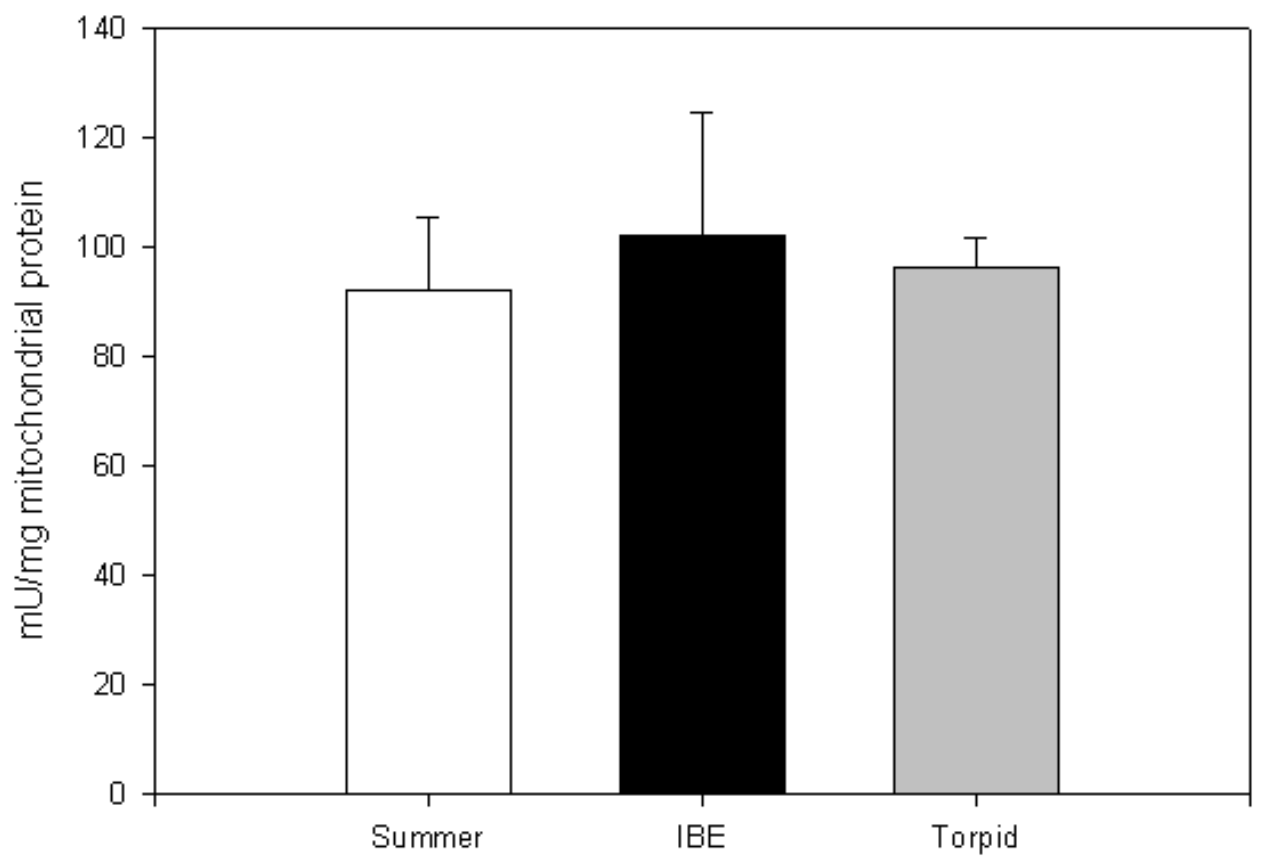

Figure 3.11 Carnitine palmitoyl transferase activity among metabolic states. The activity of carnitine-palmitoyl transferase was assessed in liver mitochondria isolated from summer, interbout euthermic (IBE) and torpid animals. Data are means $\pm \mathrm{SEM}$. One-way ANOVA and a post hoc least square difference test found there was no significant difference among metabolic states (Summer $n=6, \operatorname{IBE} n=5$, torpid $n=6$ ). 


\section{CHAPTER 4}

\section{DISCUSSION}

\subsection{Palmitoyl CoA and butylmalonate cause suppression of succinate-fuelled respiration rates.}

Palmitoyl CoA abruptly suppressed succinate-fuelled state 3 respiration by 70 $80 \%$ (relative to paired control values) in both torpid and IBE mitochondria, while butylmalonate, a well documented inhibitor of the DCT (Johnson and Chappell, 1973; Robinson and Chappell, 1967; Robinson and Williams, 1970), only inhibited respiration by $30-40 \%$ (figure 3.9 ). Inhibition of the DCT by butylmalonate confirms that inhibition of succinate transport into the mitochondrial matrix is indeed a mechanism capable of significantly suppressing mitochondrial respiration, comparable to the effect seen in torpid hibernators (44\%; figure 3.3). However, contrary to my prediction, inhibition of respiration was observed in liver mitochondria isolated from both IBE, and torpid ground squirrels (figure 3.9). If respiratory suppression in torpor is indeed a reflection of inhibition of succinate transport at the DCT, then I predicted that the DCT of torpid mitochondria would have already been significantly inhibited, and therefore less susceptible to further inhibition by butylmalonate.

Similarly, and once again contrasting to my initial prediction, the suppression of respiration by palmitoyl CoA was observed in both IBE as well as torpid mitochondria to comparable degrees. If palmitoyl CoA was solely inhibiting succinate transport, I predicted that respiration rates in both torpor and IBE would be inhibited to the same

level. However, respiration rates of IBE mitochondria were still significantly higher than those of torpid mitochondria following treatment with palmitoyl CoA (figure 3.8). If 
torpid mitochondria were already be inhibited by long chain acyl CoAs, I would have expected them to be less susceptible to further additions of palmitoyl CoA.

Although palmitoyl CoA significantly suppressed mitochondrial respiration, the extent of suppression observed exceeds the maximal suppression obtained from the synthetic DCT inhibitor butylmalonate (figure 3.9). This raises the possibility that palmitoyl CoA may be suppressing respiration via some other mechanism, either in addition to, or instead of, inhibition of succinate transport at the DCT. Although palmitoyl CoA's inhibition of the DCT has been substantiated by swelling studies, confirming that it causes inhibition of the DCT (Ventura et al., 2005), long chain-acyl CoAs have also been shown to inhibit the adenine nucleotide translocator (ANT, Lerner et al., 1972; Pande and Blanchaer, 1971), which is responsible for the exchange of ADP and ATP across the IMM. Inhibition of the ANT would effect a shortage of ADP in the mitochondrial matrix, limiting the activity of ATP synthase, and effectively impeding respiration. Inhibition of both the DCT as well as the ANT could explain the greater extent of suppression than would be expected if only the DCT was inhibited (for summary see figure 4.1).

In order to confirm the involvement of the DCT, I attempted mitochondrial swelling studies to measure the exchange rate of succinate for $\mathrm{P}_{\mathrm{i}}$, to determine if there is a difference in the activity of the DCT between IBE and torpid mitochondria. Similar studies were successfully performed in rats by Ventura et al. (2005), and in our own lab in sparrow liver mitochondria. However, when I attempted this technique on 13-lined ground squirrels liver mitochondria the measurements were not successful, and I had an 


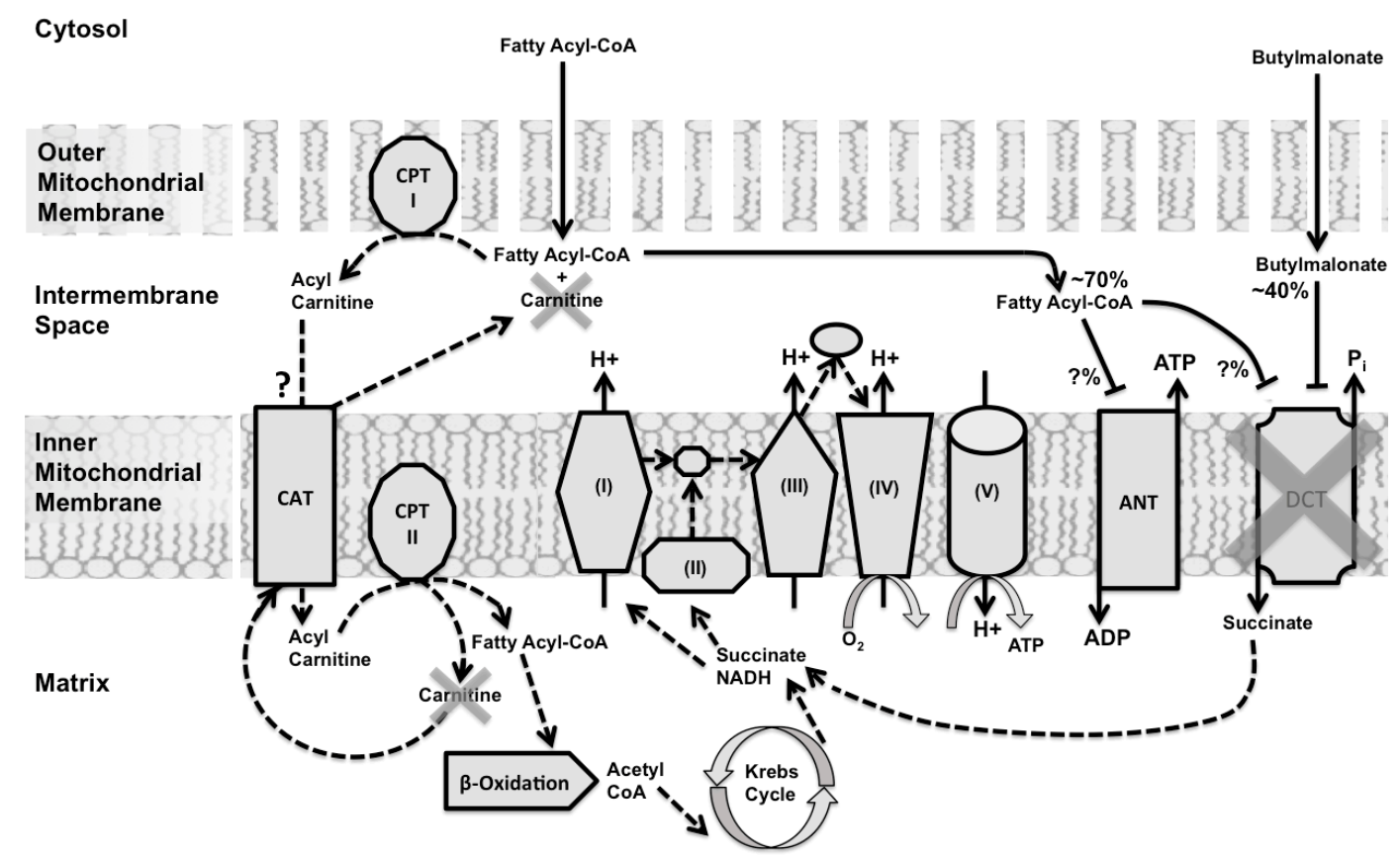

Figure 4.1 Adjusted model of the proposed effect of fatty acyl-CoAs and succinate transport on mitochondrial respiration. Butylmalonate, a known inhibitor of the dicarboxylate transporter (DCT) caused a $\sim 40 \%$ suppression of succinate-fuelled respiration in both torpid and IBE mitochondria, while treatment with a fatty acyl CoA caused a $\sim 70 \%$ suppression. As fatty acyl-CoAs are capable of inhibition of both the DCT and the adenine nucleotide transporter (ANT), both of which could contribute to decreased respiration, therefore the percent attributable to each remains unknown. The addition of carnitine following treatment with fatty acyl-CoAs did not increase respiration, despite confirmed activity of carnitine palmitoyl transferase (CPT I). Dashed lines indicate inhibited processes, solid lines indicate operating processes. 
insufficient quantity of mitochondria to perfect the technique. As a result I cannot assess how much of the total respiratory suppression caused by palmitoyl CoA is occurring at the DCT and how much suppression is occurring elsewhere, such as the ANT. Ventura et al. (2005) found that, while butylmalonate caused a $\sim 50 \%$ reduction in the function of the DCT in rat mitochondria, treatment with palmitoyl CoA effected only a $\sim 25 \%$ reduction. I could then speculate that palmitoyl CoA is only about half as effective at inhibiting the DCT as butylmalonate. Applying this to my respiration data $(\sim 40 \%$ and $\sim 70 \%$ suppression by butylmalonate and palmitoyl CoA respectively), suggests that palmitoyl CoA likely contributes a smaller proportion of suppression at the DCT than butylmalonate ( $>40 \%$ ), while the rest of suppression is contributed at the ANT or elsewhere (see figure 4.1 for summary).

\subsection{Carnitine does not reverse respiratory suppression in either torpid or palmitoyl}

\section{CoA treated mitochondria.}

Although palmitoyl CoA inhibited mitochondrial respiration, the addition of carnitine to mitochondria treated with palmitoyl CoA did not have a 'rescuing' effect on respiration as predicted (figure 3.9), nor did carnitine significantly increase respiration in torpid mitochondria (figure 3.10). I predicted that the addition of carnitine would facilitate fatty acyl CoAs transported to the matrix, relieving any accumulation of fatty acyl CoAs in the IMS that might inhibit the DCT and, as a result, succinate transport would resume and respiration would increase (figure 1.4). This prediction was further supported by the work of Nelson et al. (2009) who found carnitine concentrations in liver tissue to be higher in arousing and IBE animals, but low in torpid hibernators. However as their work was performed in homogenized liver tissue (Nelson et al., 2009), I am 
unable to distinguish whether these higher carnitine concentrations in IBE occur in the mitochondria or in the cytosol.

However, Pande and Blanchaer (1971), and Lerner et al. (1972), independently reported that inhibition of the mitochondrial ANT by palmitoyl CoA in rat heart mitochondria could be largely reversed by the addition of carnitine. This, they suggested, was a result of palmitoyl CoA transport into the matrix; a mechanism that, if operating as suggested, may have similarly reversed any inhibition of the DCT caused by palmitoyl CoA (figure 1.4). These studies also confirm that in non-hibernating rodents (Lerner et al., 1972; Pande and Blanchaer, 1971), carnitine is able to enter the IMS of isolated mitochondria. However it appears that this same mechanism cannot be applied to the inhibition of respiration affected by palmitoyl CoA in hibernator liver mitochondria.

Carnitine's failure to reverse the respiratory suppression resulting from treatment with palmitoyl CoA, suggested the possibility that the activity of CPT may be suppressed in hibernators, thus inhibiting fatty acyl CoA transport into the matrix. Composition of the IMM changes as hibernators transition between metabolic states (Armstrong et al., 2011; Chung et al., 2011), and as a membrane bound protein, CPT could be affected. If CPT activity decreases solely due to membrane remodelling, the presence of carnitine to intact mitochondria would have little effect, as low CPT activity would decrease the rate of formation of palmitoyl carnitine (figure 1.4). However total CPT activity did not differ significantly among summer, IBE or torpid animals (figure 3.11), thus eliminating the possibility that inhibition of CPT was limiting the movement of palmitoyl CoA into the matrix. 
One possibility as to why carnitine was unable to reverse the suppression caused by palmitoyl CoA is that the experimental system did not permit carnitine adequate time to eliminate palmitoyl CoA from the IMS. In hibernators, arousal from a bout of torpor occurs on the scale of hours (Staples and Brown, 2008), however purified mitochondria respiring in vitro at $37^{\circ} \mathrm{C}$ degrade over time, and as a result, experiments must be completed within approximately one hour. From the time carnitine was added to the respirometry chambers, $\sim 4$ minutes elapsed before respiration rate was recorded (see figure 2.2 for a representative trace of the sequence and timing of additions to the oxygraph chamber). The time scale, and concentrations of palmitoyl CoA and carnitine used in this experiment were comparable to those of previous work on suppression of respiration by palmitoyl CoA and its reversal by carnitine in rat liver mitochondria (Pande and Blanchaer, 1971; Wood et al., 1977). However it is possible that in 13-lined ground squirrels the 'rescuing' effect of carnitine on mitochondrial respiration may require more time, on the scale of hours, and were therefore not observed in this experiment. Wood et al. (1977) observed increasing palmitoyl CoA concentration (in the presence of carnitine) positively correlated with an increasing delay in the onset of state 3 respiration of liver mitochondria in rats. Therefore, as a saturating palmitoyl CoA concentration was used in this experiment, the correlation suggested by Wood et al. (1977) would introduce a significant delay in the onset of state 3 respiration rate, which may have been missed by the time limit imposed by this experimental system.

A second explanation as to why carnitine was unable to reverse suppression of respiration may be due to the physical structure of a fatty acyl CoA. Fatty acyl CoAs are comprised of a polar adenosine-phosphate head group attached to a lipophilic long-chain 
fatty acid tail. Duszynski and Wojtczak (1974) speculated that the lipophilic hydrocarbon tail of fatty acyl CoAs embed themselves into the mitochondrial membrane, acting as an anchor, leaving the polar adenine-phosphate head group free to competitively inhibiting mitochondrial transporters. This speculation may explain why, despite the disruption caused by physically isolating mitochondria from tissue, suppression due to fatty acyl CoAs would persist in purified mitochondrial samples, supporting the original hypothesis. However the other implication this may have is that once suppression by fatty acyl CoAs occurs (in this case by addition of palmitoyl CoA), reversal is difficult due to the anchoring effect of the fatty acid tails, and even in the presence of carnitine their affinity for the mitochondrial membrane might limit association with CPT and therefore prevent subsequent transport into the matrix by CAT. However, that being said, hibernators survive the entire winter on endogenous fat stores, which in order to be used must be converted to fatty acyl CoAs and pass through the IMM on their way to $\beta$ oxidation in the matrix; implying that affiliation with the mitochondrial membrane is not entirely irreversible, but again, may occur more slowly than could be observed by the current experiment.

\subsection{Liver mitochondrial respiration in torpid, interbout euthermic (IBE) and summer 13-lined ground squirrels.}

The liver alone does not account for all of the metabolic suppression observed in hibernators ( $\sim 95 \%$; Geiser and Ruf, 1995). However it consumes $\sim 20 \%$ of total inspired oxygen (in rates; Rolfe and Brown, 1997), and I showed that succinate-fuelled liver mitochondrial respiration was suppressed by $44 \%$ in torpor relative to $\operatorname{IBE}\left(37^{\circ} \mathrm{C}\right.$; figure 3.3), suggesting that active suppression in the liver could accounts for a $8.8 \%$ reduction in 
whole animal oxygen consumption, with no change in $T_{b}$. During entrance into a torpor bout whole animal oxygen consumption declines before $T_{b}$ (reviewed in Staples and Brown, 2008), so mitochondrial suppression in metabolically important tissues, such as liver, may be especially important during the early stages of entrance. As entrance progresses, the passive thermal effects of lowering $\mathrm{T}_{\mathrm{b}}$ from $37^{\circ} \mathrm{C}$ to $5^{\circ} \mathrm{C}$ further decrease metabolism in these tissues. Regardless of the contribution to whole-animal metabolism in hibernators, the suppression of succinate-fuelled respiration in liver mitochondria happens rapidly and reversibly in ground squirrels, offering a good model to study the plasticity and regulation of mitochondrial metabolism.

As in previous studies, succinate-fuelled state 3 respiration rates of isolated torpid mitochondria were significantly suppressed compared to mitochondria from summer and IBE animals (Armstrong and Staples, 2010; Barger et al., 2003; Brown et al., 2012; Chung et al., 2011; Muleme et al., 2006; Pehowich and Wang, 1984). State 4 respiration rates were used to calculate the respiratory control ratio (RCR; state 3 /state 4), an indication of how well coupled substrate oxidation is to ATP synthesis, which generally relates to the integrity of the IMM. Mean RCRs for this study were 2.82 in torpor, 3.23 in IBE and 3.67 in summer animals; which are comparable to RCRs of previous studies (Armstrong and Staples, 2010; Brown et al., 2012), and indicate well coupled mitochondria.

Traditionally, mitochondrial respiration rates are expressed relative to total mitochondrial protein (figure 3.3). However, if the concentrations of mitochondrial proteins that do not contribute to oxidative phosphorylation, such enzymes of the urea cycle, change among metabolic states, the respiration rates expressed this way will 
change as well. Guderley et al. (2005) explored alternative methods of expressing mitochondrial respiration by standardizing rates relative to mitochondrial content of phospholipids, transporters and ETC cytochromes . In the current study, mitochondrial respiration rate was calculated relative to the activity of cytochrome c oxidase (ETC complex IV, figure 3.5), to provide an independent means of assessing respiration. This method showed torpid mitochondria to be significantly suppressed (figure 3.5), which is consistent with the respiration rates calculated relative to total mitochondrial protein (figure 3.3). I can conclude that suppression of respiration in torpor is not attributable to a change in the relative abundance of mitochondrial proteins and, therefore, that expressing respiration relative to total mitochondrial protein remains an appropriate method.

A second concern was that the any differences in respiration rate among metabolic states were due to differences in the relative abundance of different ETC proteins. Western blot analysis showed that although the abundance of ETC complex I was higher in IBE than torpor or summer animals, all other ETC complexes (II-V) remained consistent among metabolic states (figure 3.6). In this study, all rates of respiration recorded were succinate fuelled, and in the presence of rotenone (ETC complex I inhibitor). Therefore the ETC complexes II - IV, contributing to the respiration rates I measured, did not differ significantly among metabolic states, implying that the metabolic suppression observed in torpor is not attributable to a changes in ETC protein densities, but rather a regulated change in oxidative phosphorylation.

\subsection{Conclusions and future directions.}

A principal limitation imposed by this experimental system was small sample size. There were a limited number of animals in our colony, as they are obtained from the 
wild, and capture is limited to a short time period, placing a constraint on the number of biological replicates for all experiments performed in this thesis. Furthermore sampling hibernating animals imposed timing constraints, as data could only be collected seasonally, as the hibernation season is limited to October-March and animals do not hibernate in summer months. The spontaneous nature of IBE further added to the strict limitations as to when an animal could be sampled. Additionally there is a limitation to the quantity of mitochondria that can be purified from the liver due to its small size $(\sim 5 \mathrm{~g})$, and mitochondrial purification, while decreasing contamination of samples, decreases the overall mitochondrial yield. Overall this places a physical limit on the number of experiments that can be performed on a single biological replicate. Finally, and arguably one of the most important limitations to be considered, is the viability of purified mitochondria over time. In the purification process mitochondria are physically homogenized from living tissue, and maintained on ice suspended in a buffer made to approximate the conditions in the cell. In these conditions the mitochondria only remain viable for a limited time (on the scale of hours). Once introduced to the oxygraph, where their respiration rate is measured at $37^{\circ} \mathrm{C}$ while undergoing constant turbulence due to stirring, the mitochondria's viability is limited to $\sim 45$ minutes. These strict time limitations impose serious constraints to experimental design. In biological systems palmitoyl CoA may be cleared from the inner mitochondrial membrane by carnitine on the scale of hours, however in this system I was only able to observe a period on the scale of minutes, which does not allow for a full exploration of the proposed model.

Inhibition of succinate transport by butylmalonate suggests that inhibition of the DCT has the potential to suppress succinate fuelled state 3 respiration. There is no doubt 
that palmitoyl CoA is a potent inhibitor of mitochondrial respiration, capable of inhibiting succinate-fuelled respiration. However this suppression may not be solely related to inhibition of succinate transport into the matrix by the DCT, and is likely causing respiratory suppression by inhibiting additional mitochondrial transporters such as the ANT. Regardless, the unexpected inhibition of already suppressed torpid mitochondria, by both butylmalonate and palmitoyl CoA, suggests that inhibition of succinate transport at the DCT is likely not the central mechanism operating to suppress respiration during torpor. The data instead suggest that such a mechanism likely occurs downstream of succinate transport at the DCT. Carnitine had no rescuing effect on torpid mitochondria, nor on mitochondria suppressed by treatment with palmitoyl CoA, despite confirmed activity of CPT, suggesting that fatty acyl CoAs were not being removed from the IMS (for summary see figure 4.1).

Butylmalonate is a competitive inhibitor of the DCT (Coty and Pedersen, 1974; Crompton et al., 1974; Robinson and Chappell, 1967) caused only partial inhibition of succinate oxidation ( $\sim 40 \%$; figure 3.9). This partial inhibition may have resulted because the concentration of succinate in this design was very high $(10 \mu \mathrm{M})$. Competitive inhibitors may increase the $\mathrm{K}_{\mathrm{m}}$ (substrate concentration at which the reaction rate is at $50 \%$ of the maximum reaction rate, $\mathrm{V}_{\max }$ ) of the substrate, however the $\mathrm{V}_{\max }$ does not change (Chou, 1976). Therefore, with a high enough substrate concentration, in this case succinate, the effects of an inhibitor may be diminished. Although the physiological concentration of succinate during in vivo mitochondrial metabolism is not known, it is likely much lower than $10 \mu \mathrm{M}$ (Brown et al., 2013). Therefore I feel it would be 
interesting to determine if lower concentrations of succinate would permit greater inhibition of the DCT by butylmalonate.

To clarify the involvement of the DCT in the inhibition of mitochondrial respiration by long chain acyl CoAs, the effect of long chain acyl CoAs on glutamatefuelled state 3 respiration should be investigated. If long chain acyl CoAs differentially suppress glutamate-fuelled respiration rates relative to succinate-fuelled respiration rates, then it could be concluded that some inhibition of succinate transport at the DCT is occurring, (as succinate is solely transported by the DCT, and glutamate by multiple independent transporters). However if suppression is relatively similar, it would indicate that the effects of long chain acyl CoAs on respiration does not involve the DCT.

Despite the observation that inhibition of succinate transport at the DCT is not the principle mechanism behind the suppression observed in torpid hibernators, other potential roles for fatty acyl CoAs in hibernation have yet to be sufficiently explored. Inhibition of the ANT by fatty acyl CoAs in non-hibernators is well established in the literature, as well as its potential for the reversal of this suppression by carnitine (Lerner et al., 1972; Pande and Blanchaer, 1971; Shug et al., 1971; Wood et al., 1977). As transport of ADP into the mitochondrial matrix by ANT is a requirement for ATP synthesis, inhibition of the ANT would cause a suppression of respiration, similar to that seen in torpor, suggesting a physiological role for long chain fatty acyl CoAs in the regulation of mitochondrial respiration in hibernation.

One other potential direction for this research, is to design an experiment that examines a longer period of time following additions of carnitine, which may provide adequate time for 1) dissociation of fatty acyl CoAs from the mitochondrial membrane, 
and 2) the action of CPT and CAT to remove fatty acyl CoA from the IMS. Another avenue to determine why carnitine was unable to reverse suppression induced by palmitoyl CoAs would be to determine the activity of CAT, which could be inhibited in torpor, preventing movement of fatty acyl CoAs in the matrix. Finally, although unsuccessfully attempted in this study, completion of mitochondrial swelling studies in hibernators to examine the activity of succinate transport at the DCT among different metabolic states could provide an excellent insight into whether further research into inhibition of the DCT is warranted. 


\section{REFERNCES}

Armitage, K. B., Downhower, J. F. and Svendsen, G. E. (2013). Seasonal Changes in Weights of Marmots. American Midland Naturalist 96, 36-51.

Armstrong, C. and Staples, J. F. (2010). The role of succinate dehydrogenase and oxaloacetate in metabolic suppression during hibernation and arousal. Journal of comparative physiology. B, Biochemical, systemic, and environmental physiology 180, 775-83.

Armstrong, C., Thomas, R. H., Price, E. R., Guglielmo, C. G. and Staples, J. F. (2011). Remodeling Mitochondrial Membranes during Arousal from Hibernation. Physiological and Biochemical Zoology 84, 438-449.

Barger, J. L., Brand, M. D., Barnes, B. M. and Boyer, B. B. (2003). Tissue-specific depression of mitochondrial proton leak and substrate oxidation in hibernating arctic ground squirrels. American journal of physiology. Regulatory, integrative and comparative physiology 284, R1306-13.

Bennett, A. F. and Ruben, J. A. (1979). Endothermy and Activity in Vertebrates. Science 206, 649-654.

Bernardi, P., Krauskopf, A., Basso, E., Petronilli, V., Blachly-Dyson, E., BlalchyDyson, E., Di Lisa, F. and Forte, M. a (2006). The mitochondrial permeability transition from in vitro artifact to disease target. The FEBS journal 273, 2077-99.

Bradford, M. M. (1976). A rapid and sensitive method for the quantitation of microgram quantities of protein utilizing the principle of protein-dye binding. Analytical biochemistry 72, 248-54.

Brown, J. C. L., Chung, D. J., Belgrave, K. R. and Staples, J. F. (2012).

Mitochondrial metabolic suppression and reactive oxygen species production in liver and skeletal muscle of hibernating thirteen-lined ground squirrels. American journal of physiology. Regulatory, integrative and comparative physiology 302, R15-28.

Brown, J. C. L., Chung, D. J., Cooper, A. N. and Staples, J. F. (2013). Regulation of succinate-fuelled mitochondrial respiration in liver and skeletal muscle of hibernating thirteen-lined ground squirrels. The Journal of experimental biology 216, 1736-43.

Buck, C. L. and Barnes, B. M. (2000). Effects of ambient temperature on metabolic rate , respiratory quotient, and torpor in an arctic hibernator. American journal of physiology. Regulatory, integrative and comparative physiology 279, R255-R262. 
Carey, H. V, Andrews, M. T. and Martin, S. L. (2003). Mammalian hibernation: cellular and molecular responses to depressed metabolism and low temperature. Physiological Reviews 83, 1153-1181.

Casey, T. M. (1981). Nest Insulation: Energy Savings to Brown Lemmings Using a Winter Nest. Oeologia 50, 199-204.

Chou, T.-C. (1976). Derivation and properties of Michaelis-Menten type and Hill type equations for reference ligands. Journal of Theoretical Biology 59, 253-254.

Chung, D. J. (2013). Changes in the Mitochondrial Phosphoproteome during Mammalian Hibernation. 34-36.

Chung, D., Lloyd, G. P., Thomas, R. H., Guglielmo, C. G. and Staples, J. F. (2011). Mitochondrial respiration and succinate dehydrogenase are suppressed early during entrance into a hibernation bout, but membrane remodeling is only transient. Journal of comparative physiology. B, Biochemical, systemic, and environmental physiology 181, 699-711.

Chung, D. J., Szyszka, B., Brown, J. C. L., Hüner, N. P. A. and Staples, J. F. (2013). Changes in the mitochondrial phosphoproteome during mammalian hibernation. Physiological genomics 45, 389-399.

Coty, W. A. and Pedersen, P. L. (1974). Phosphate Transport in Rat Liver Mitochondria. The Journal of biological chemistry 249, 2593-2598.

Coty, W. and Pendersen, P. (1975). Phosphate transport in rat liver mitochondria. Molecular and Cellular Biochemistry 9, 109-124.

Crompton, M., Palmieri, F., Capano, M. and Quagliariello, E. (1974). The transport of sulphate and sulphite in rat liver mitochondria. The Biochemical journal 142, $127-37$.

Daan, S., Barnes, B. M. and Strijkstra, A. M. (1991). Warming up for sleep? Ground squirrels sleep during arousals from hibernation. Neuroscience Letters 128, 265268.

Duszyinski, J. and Wojtczak, L. (1974). Effect of detergents and ADP translocation in Mitochondria. FEBS Letter 40, 72-76.

Florant, G. L. and Heller, H. C. (1977). CNS regulation of body temperature in euthermic and hibernating marmots (Marmota flaviventris). American Journal of Physiology 232, R203-R208. 
Florant, G. L., Nuttle, L. C., Mullinex, D. E. and Rintoul, D. A. (1990). Plasma and white adipose tissue lipid composition in marmots. American Journal of Physiology Regulatory, Integrative and Comparative Physiology 258, R1123-R1131.

Fritz, I. and Marquis, N. (1965). The role of acylcarnitine esters and carnitine palmityltransferase in the transport of fatty acyl groups across mitochondrial membranes. Proceedings of the National Acadamy of Science USA 54, 1226-1233.

Geiser, F. (2004). Metabolic rate and body temperature reduction during hibernation and daily torpor. Annual review of physiology 66, 239-74.

Geiser, F. and Ruf, T. (1995). Hibernation versus daily torpor in mammals and birds : Physiological variables and classification of torpor patterns. Physiological Zoology 68, 935-966.

Gnaiger, F. (1983). Calculation of equilibrium oxygen concentration. In: Polarographic oxygen sensors: aquatic and physiological applications. (ed. Gnaiger, F.) Berlin, Heidelberg, New York: Springer.

Guderley, H., Turner, N., Else, P. L. and Hulbert, A. J. (2005). Why are some mitochondria more powerful than others: Insights from comparisons of muscle mitochondria from three terrestrial vertebrates. Comparative Biochemistry and Physiology, Part B 142, 172-180.

Hart, J. S. (1956). Seasonal Changes in Insulation of the Fur. Canadian Journal of Zoology 34, 53-57.

Hatefi, Y. (1985). The mitochondrial electron transport and oxidative phosphorylation system. Annual review of biochemistry 54, 1015-1069.

Heldmaier, G., Ortmann, S. and Körtner, G. (1993). Energy requirement of hibernating alpine marmots. In Life in the Cold-Ecological, Physiological, and Molecular Mechanisms. (ed. Carey, C., Florant, G. L., Wunder, B. A., and Horwitz, B.), pp. 175-183. Boulder: Westview Press.

Heldmaier, G., Ortmann, S. and Elvert, R. (2004). Natural hypometabolism during hibernation and daily torpor in mammals. Respiratory physiology \& neurobiology 141, 317-29.

Heller, H. and Hammel, H. (1972). CNS control of body temperature during hibernation. Comparative Biochemistry and Physiology 41, 349-359.

Hill, R. W., Wyse, G. A. and Anderson, M. (2008). Animal Physiology. 2nd ed. Sunderland: Sinauer Associates Inc. 
Hochachka, P. W. and Somero, G. N. (2002). Biochemical Adaptation: Mechanism and process in physiological evolution. New York: Oxford University Press.

Hoy, A. J., Bruce, C. R., Turpin, S. M., Morris, A. J., Febbraio, M. a and Watt, M. J. (2011). Adipose triglyceride lipase-null mice are resistant to high-fat diet-induced insulin resistance despite reduced energy expenditure and ectopic lipid accumulation. Endocrinology 152, 48-58.

Indiveri, C., Prezioso, G., Dierks, T., Kramer, R. and Palmieri, F. (1993). Kinetic characterization of the reconstituted dicarboxylate carrier from mitochondria: a fourbinding-site sequential transport system. Biochimica et biophysica acta 1143, 310318.

Johnson, R. and Chappell, J. (1973). The transport of inorganic phosphate by the mitochondrial dicarboxylate carrier. Biochemical Journal 134, 769-774.

Kerner, J. and Hoppel, C. (2000). Fatty acid import into mitochondria. Biochimica et biophysica acta 1486, 1-17.

Landau, B. R. and Dawe, A. R. (1958). Respiration in the hibernation of the 13-lined ground squirrel. American Journal of Physiology 194, 75-82.

LaNoue, K. F. and Schoolwerth, a C. (1979). Metabolite transport in mitochondria. Annual review of biochemistry 48, 871-922.

Lerner, E., Shug, A. L., Elson, C. and Shrago, E. (1972). Reversible Inhibition of Adenine Nucleotide Long Chain Fatty Acyl Coenzyme A Esters in Liver Mitochondria of Diabetic and Hibernating Animals. The Journal of biological chemistry 247, 1513-1519.

McGarry, J. D. and Brown, N. F. (1997). The mitochondrial carnitine palmitoyltransferase system. From concept to molecular analysis. European journal of biochemistry / FEBS 244, 1-14.

McGivan, J. and Klingenberg, M. (1971). Correlation between $\mathrm{H}+$ and anion movement in mitochondria and the key role of the phosphate carrier. European Journal of Biochemistry 20, 392-399.

Mckeever, S. (1963). Seasonal Changes in Body Weight, Reproductive Organs, Pituitary, Adrenal Glands, Thyroid Gland, and Spleen of the Belding Ground Squirrel (Citellus Beldingi). The American journal of anatomy 113, 153-73.

Muleme, H. M., Walpole, A. C. and Staples, J. F. (2006). Mitochondrial metabolism in hibernation: metabolic suppression, temperature effects, and substrate preferences. Physiological and biochemical zoology 79, 474-83. 
Nelson, C. J., Otis, J. P., Martin, S. L. and Carey, H. V (2009). Analysis of the hibernation cycle using LC-MS-based metabolomics in ground squirrel liver. Physiological genomics 37, 43-51.

Palmieri, F. (2004). The mitochondrial transporter family (SLC25): physiological and pathological implications. European journal of physiology 447, 689-709.

Pande, S. V (1975). A mitochondrial carnitine acylcarnitine translocase system. Proceedings of the National Academy of Sciences of the United States of America 72, 883-7.

Pande, S. V. and Blanchaer, M. C. (1971). Reversible Inhibition of Mitochondrial Phosphorylation by Long Chain Acyl Coenzyme A Esters. The Journal of biological chemistry 246, 402-411.

Pehowich, D. J. and Wang, L. C. H. (1984). Seasonal changes in mitochondrial succinate dehydrogenase activity in a hibernator,Spermophilus richardsonii. Journal of Comparative Physiology B 154, 495-501.

Petit, P. X., O'Connor, J. E., Grunwald, D. and Brown, S. C. (1990). Analysis of the membrane potential of rat- and mouse-liver mitochondria by flow cytometry and possible applications. The Federation of European Biochemical Societies Journal 194, 389-397.

Prendergast, B. J., Freeman, D. a, Zucker, I. and Nelson, R. J. (2002). Periodic arousal from hibernation is necessary for initiation of immune responses in ground squirrels. American journal of physiology. Regulatory, integrative and comparative physiology 282, R1054-62.

Rector, R. S., Uptergrove, G. M., Morris, E. M., Borengasser, S. J., Laughlin, M. H., Booth, F. W., Thyfault, J. P. and Ibdah, J. a (2011). Daily exercise vs. caloric restriction for prevention of nonalcoholic fatty liver disease in the OLETF rat model. American journal of physiology. Gastrointestinal and liver physiology 300, G87483.

Robinson, B. and Chappell, J. (1967). The Inhibition of Malate, Tricarboxylate and oxoglutarate entry into mitochondria by 2-n-butylmalonate. Biochemical and biophysical research communications 28, 249-255.

Robinson, B. and Williams, G. (1970). The sensitivity of dicarboxylate anion exchange reactions to transport inhibitors in rat-liver mitochondira. Biochimica et Biophysica Acta 216, 63-70.

Rolfe, D. F. S. and Brown, G. C. (1997). Cellular Energy Utilization of Standard Metabolic and Molecular Origin Rate in Mammals. Physiological Reviews 77, 731758. 
Rostovtseva, T. K. and Bezrukov, S. M. (2008). VDAC regulation: role of cytosolic proteins and mitochondrial lipids. Journal of bioenergetics and biomembranes $\mathbf{4 0 ,}$ 163-70.

Shrago, E., Woldegiorgis, G., Ruoho, A. E. and DiRusso, C. C. (1995). Fatty Acyl CoA Esters as Regulators of Cell Metabolism. Prostaglandins Leukotrienes and Essential Fatty Acids 52, 163-166.

Shug, A., Lerner, E., Elson, C. and Shrago, E. (1971). The inhibition of adenine nucleotide translocase activity by oleoyl $\mathrm{CoA}$ and its reversal in rat liver mitochondria. Biochemical and biophysical research communications 43, 557-563.

Smith, C. C. and Reichman, O. J. (1984). The Evolution of Food Caching by Bird and Mammals. Annual Review of Ecology and Systematics 15, 329-351.

Srere, H. K., Wang, L. C. and Martin, S. L. (1992). Central role for differential gene expression in mammalian hibernation. Proceedings of the National Academy of Sciences of the United States of America 89, 7119-23.

Staples, J. F. and Brown, J. C. L. (2008). Mitochondrial metabolism in hibernation and daily torpor: a review. Journal of comparative physiology. B, Biochemical, systemic, and environmental physiology 178, 811-27.

Takaki, M., Nakahara, H., Kawatani, Y., Utsumi, K. and Suga, H. (1997). No suppression of respiratory function of mitochondrial isolated from the hearts of anesthetized rats with high-dose pentobarbital sodium. The Japanese journal of physiology 47, 87-92.

Tøien, Ø., Blake, J., Edgar, D. M., Grahn, D. a, Heller, H. C. and Barnes, B. M. (2011). Hibernation in black bears: independence of metabolic suppression from body temperature. Science 331, 906-9.

Vaughan, D. K., Gruber, A. R., Michalski, M. L., Seidling, J. and Schlink, S. (2006). Capture, care, and captive breeding of 13-lined ground squirrels, Spermophilus tridecemlineatus. Lab Animal 35, 33-40.

Ventura, F. V, Ruiter, J. P. N., Ijlst, L., Almeida, I. T. and Wanders, R. J. A. (1995). Inhibition of oxidative phosphorylation by palmitoyl-CoA in digitonin permeabilized fibroblasts: implications for long-chain fatty acids beta-oxidation disorders. Biochimica et Biophysica 1272, 14-20.

Ventura, F. V, Ruiter, J., Ijlst, L., De Almeida, I. T. and Wanders, R. J. a (2005). Differential inhibitory effect of long-chain acyl-CoA esters on succinate and glutamate transport into rat liver mitochondria and its possible implications for longchain fatty acid oxidation defects. Molecular genetics and metabolism 86, 344-52. 
Wang, L. C. H. (1978). Energetics and field aspects of mammalian torpor: the Richardson's ground squirrel. In Strategies in Cold: Natural Torpidity and Thermogenesis. (ed. Hudson, J. W. and Wang, L. C. H.), pp. 109-145. London: Academic Press.

Wang, L. (1979). Time patterns and metabolic rates of natural torpor in the Richardson's ground squirrel. Canadian Journal of Zoology 57, 149-155.

White, C. R., Phillips, N. F. and Seymour, R. S. (2006). The scaling and temperature dependence of vertebrate metabolism. Biology Letters 2, 125-127.

Wojtczak, L. (1976). Effect of Long-Chain Fatty Acids and Acyl-CoA on Mitochondrial Permiability, Transport, and Energy-Coupling Processes. Journal of Bioenergetics and Biomembranes 8, 293-311.

Wojtczak, L. and Zaluska, H. (1967). The inhibition of translocation of adenine nucleotides through mitochondrial membranes by oleate. Biochemical and biophysical research communications 28, 76-81.

Woldegiorgis, G., Yousufzai, S. Y. K. and Shrago, E. (1982). Studies on the Interaction of Palmitoyl Coenzyme A with the Adenine Nucleotide Translocase. The Journal of biological chemistry 257, 14783-14787.

Wood, J. M., Wallick, E. T., Schwartz, A. and Chang, C.-H. (1977). The Effect of Palmitoyl-Coenzyme A on Rat Heart and Liver Mitochondria. Biochimica et biophysica acta 486, 331-340.

Xu, R., Andres-Mateos, E., Mejias, R., MacDonald, E. M., Leinwand, L. A., Merriman, D. K., Fink, R. H. A. and Cohn, R. D. (2013). Hibernating squirrel muscle activates the endurance exercise pathway despite prolonged immobilization. Experimental Neurology Article in Press.

Young, R. A. (1976). Fat, Energy and Mammalian Survival. American Zoologist 16, 699-710. 


\section{Appendix A)}

\section{Figure A.1 Animal use ethics approval Western : :}

AUP Number: 2012-016

PI Name: Staples, Jim

AUP Title: Regulation Of Mitochondrial Metabolism In Mammalian Hibernation And Ageing.

Approval Date: 07/13/2012

Official Notice of Animal Use Subcommittee (AUS) Approval: Your new Animal Use Protocol (AUP) entitled "Regulation Of Mitochondrial Metabolism In Mammalian Hibernation And Ageing." has been APPROVED by the Animal Use Subcommittee of the University Council on Animal Care. This approval, although valid for four years, and is subject to annual Protocol Renewal.2012-016::1

1. This AUP number must be indicated when ordering animals for this project.

2. Animals for other projects may not be ordered under this AUP number.

3. Purchases of animals other than through this system must be cleared through the ACVS office. Health certificates will be required.

The holder of this Animal Use Protocol is responsible to ensure that all associated safety components (biosafety, radiation safety, general laboratory safety) comply with institutional safety standards and have received all necessary approvals. Please consult directly with your institutional safety officers.

Submitted by: Copeman, Laura

on behalf of the Animal Use Subcommittee

University Council on Animal Care

The University of Western Ontario

Animal Use Subcommittee / University Council on Animal Care

Health Sciences Centre, - London, Ontario - CANADA - N6A 5C1

PH: 519-661-2111 ext. 86768 • FL 519-661-2028

Email: auspc@uwo.ca • http://www.uwo.ca/animal/website 


\section{Appendix B)}

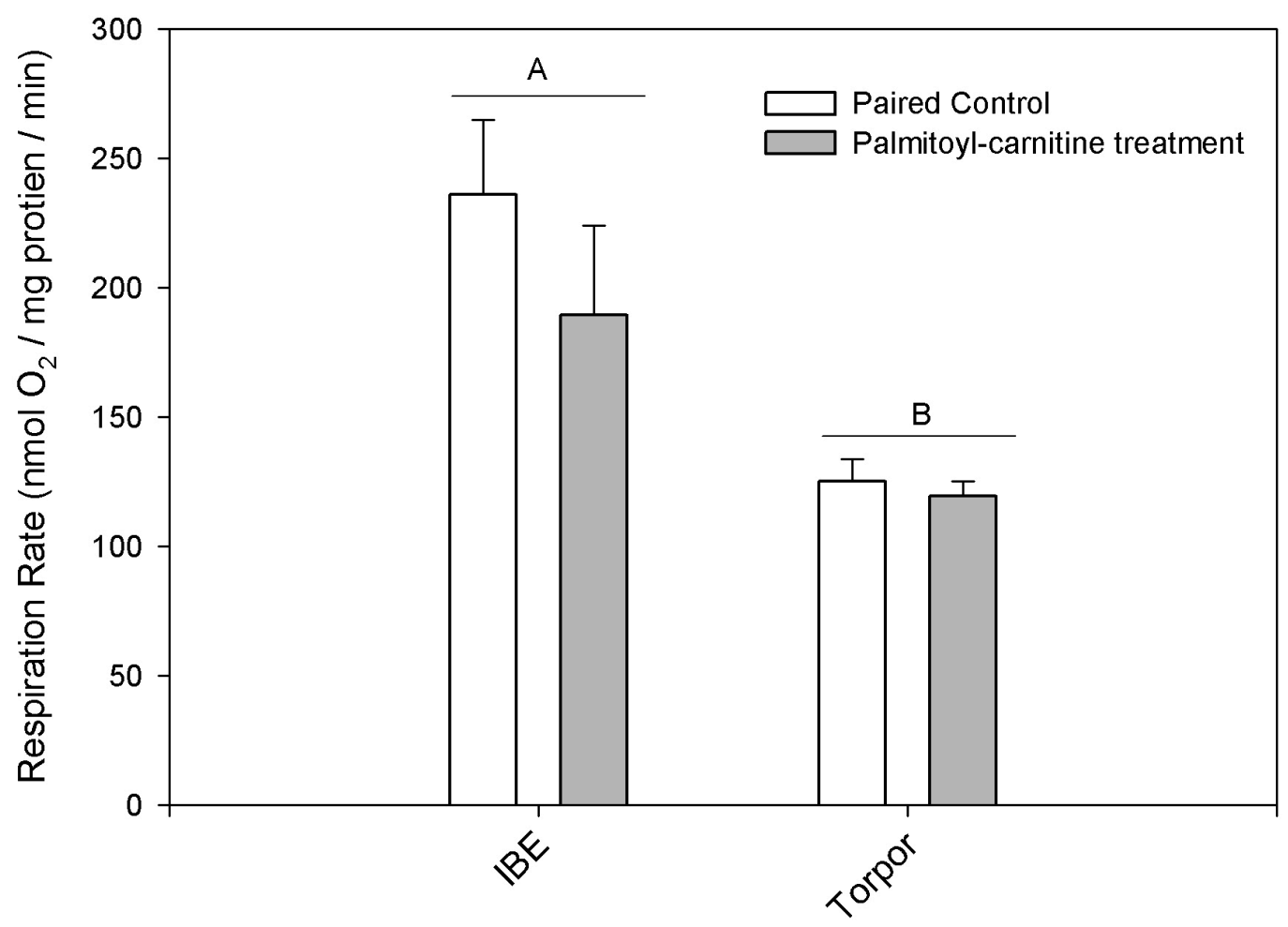

Figure B.1 Palmitoyl-carnitine has no effect on state 3 succinate fuelled mitochondrial respiration of liver mitochondria in interbout euthermic (IBE) and torpid 13-lined ground squirrels at $37^{\circ} \mathrm{C}$. Data are means $\pm \mathrm{SEM}$. Succinate fuelled state 3 respiration was established, and immediately followed by the addition of palmitoyl-carnitine. Once respiration rates reached a steady state they were recorded. A control treatment (identical to the experimental treatment, but receiving equivalent volumes of MiR05 buffer solution in place of palmitoyl-carnitine) was run in tandem with the experimental treatment. Different letters depict a significant difference of respiration rate between metabolic state $(\operatorname{IBE} n=5$, torpor $n=6)$ No significant difference $(\mathrm{P}=0.308)$ was found between treatments, indicating that palmitoyl-carnitine had no effect on mitochondrial respiration. 


\section{CURRICULUM VITAE}

Name:

Alex Cooper

Education:

MSc Biology - 2013

University of Western Ontario

BSc Biology - 2010

Award:

University of Guelph

UWO Society of Graduate Students, 2013 Graduate Student Teaching Award

\section{Peer Reviewed Articles:}

Brown J.C.L., Chung D.J., Cooper A.N., Staples J.F. 2013. Regulation of succinatefuelled mitochondrial respiration in liver and skeletal muscle of hibernating thirteen-lined ground squirrels. J. Exp. Biol. 216:1736-1743.

Brown J.C.L., Cooper A.N., Faure P.A, Staples J.F. Long-lived bats and sparrows use different mechanisms to lower muscle mitochondrial ROS production. (Submitted)

Cooper A.N., Brown J.C.L., Staples J.F.. Do fatty acyl CoAs contribute to mitochondrial suppression during torpor in hibernating ground squirrels? (In preparation)

\section{Presentations:}

Cooper A.N., Brown J.C.L., Staples J.F. 2013. The effect of palmitoyl CoA and carnitine on succinate-fuelled liver mitochondrial respiration rates in hibernating ground squirrels. Canadian Society of Zoologists Annual Meeting, Guelph, Ontario.

Brown J.C.L., Cooper A.N., Faure P.A., Staples J.F. 2013.Long-lived big brown bats (Eptesicus fuscus) and house sparrows (Passer domesticus) use different mechanisms to lower ROS production in skeletal muscle and heart mitochondria relative to short-lived house mice (Mus musculus). Canadian Society of Zoologists Annual Meeting, Guelph, Ontario. 